\title{
Tabularia
}

\section{La maîtrise d'ouvrage de Jean de France, duc de Berry (1340-1416). Reconstitution et analyse critique d'une documentation dispersée}

The building skills of Jean de France, duke of Berry (1340-1416). Reconstruction and critical analysis of a dispersed archive

\section{Thomas Rapin}

\section{OpenEdition}

\section{Journals}

\section{Édition électronique}

URL : http://journals.openedition.org/tabularia/1400

DOI : $10.4000 /$ tabularia. 1400

ISSN : 1630-7364

Éditeur :

CRAHAM - Centre Michel de Boüard, Presses universitaires de Caen

\section{Référence électronique}

Thomas Rapin, «La maîtrise d'ouvrage de Jean de France, duc de Berry (1340-1416). Reconstitution et analyse critique d'une documentation dispersée », Tabularia [En ligne], Les sources comptables, méthodologie, critique et édition, mis en ligne le 12 juillet 2006, consulté le 10 décembre 2020. URL: http://journals.openedition.org/tabularia/1400 ; DOI : https://doi.org/10.4000/tabularia.1400

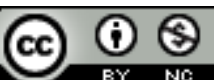

la revue Tabularia est mise à disposition selon les termes de la Licence Creative Commons Attribution - Pas d'Utilisation Commerciale 4.0 International. 


\title{
La maîtrise d'ouvrage de Jean de France, duc de Berry (1340-1416). Reconstitution et analyse critique d'une documentation dispersée
}

\author{
The building skills of Jean de France, duke of Berry \\ (1340-1416). Reconstruction and critical analysis \\ of a dispersed archive
}

\author{
Thomas RAPIN ${ }^{1}$ \\ CESCM-UMR 6580 \\ Université de Poitiers \\ thomas.rapin@libertysurf.fr
}

\section{Résumé:}

Une étude approfondie des archives de Jean de Berry peut être d'un grand secours pour appréhender le problème de sa maîtrise d'ouvrage, vaste sujet qui ne peut être simplement limité aux prestigieuses résidences - le château de Mehun-sur-Yèvre par exemple - ou à l'ambitieux programme politique de la Sainte-Chapelle de Bourges. L'étendue et la variété des chantiers exigèrent la mobilisation de tous : du simple châtelain aux plus proches officiers du prince, des artisans locaux aux maîtres d'œuvre les plus qualifiés. Cette fusion de la construction et l'entretien du bâti dans l'action du gouvernement princier explique le caractère hétéroclite, dispersé et souvent très lacunaire des archives.

Mots-clés: Jean de Berry, maîtrise d'ouvrage, maîtrise d'œuvre, architecture XIV siècle, Bourges, Riom, Poitiers.

\begin{abstract}
:
A closed study of John of Berry's archives can be usefull to understand his building mastery: a vast subject that cannot be limited to the most famous residences, e.g. the castle of Mehun-sur-Yèvre, or the ambitious political building scheme for the Sainte-Chapelle at Bourges. The number and diverse character of each building project required the mobilisation of every available man, from the modest castellan to the most important court officer, from the local craftsman to the most skilled and qualified stone mason. The construction of a building and its ensuing maintenance were closely linked. Since they formed part of the ducal administation, their interconnection explains the character of the documentary archive which is irregular, disorganised and incomplete. Keywords: John of Berry, building mastery, masson mastery, architecture of the XIV th century, Bourges, Riom, Poitiers.
\end{abstract}

1. Doctorant, CESCM, université de Poitiers et EHESS sous la direction associée de $\mathrm{M}^{\mathrm{me}}$ Claude AndraultSchmitt et de M. Jean Chapelot. Je tiens à remercier Jean et Odette Chapelot pour leur soutien et leur aide. 
Une des contributions récentes de la recherche historique est la mise en évidence du rôle politique de premier plan tenu par Jean de Berry (1340-1416) «fils, frère et oncle de roi de France $»^{2}$. Très impliqué dans les affaires du royaume, le duc partage son temps entre la vie de cour à Paris et le gouvernement de son apanage: les duchés de Berry et d'Auvergne, le comté de Poitou. Bien que son domaine, presque aussi vaste que celui de son frère Philippe, commande les voies de passages en direction de la Guyenne et du Languedoc, il reste à l'écart des grands axes commerciaux et offre un rendement somme toute très relatif ${ }^{3}$. Le cœur de l'apanage se structure principalement autour du Berry et du Poitou ; l'Auvergne reste le parent pauvre parmi les possessions du prince.

En matière de maîtrise d'ouvrage, Jean de Berry ne semble pas se départir des princes du sang, soucieux de poser les fondements d'une nouvelle dynastie dans ses terres, il lance un important programme de réfections et de constructions castrales et palatiales. Ces programmes s'inscrivent principalement dans les trente dernières années du XIVe siècle.

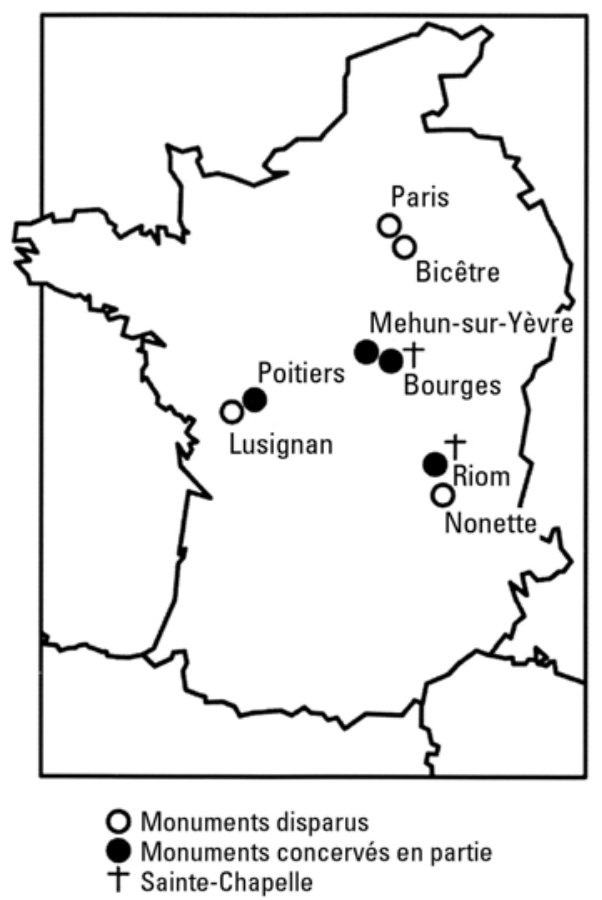

Figure 1 : Les principales résidences, palais et constructions de Jean de France, duc de Berry (1372-1416).

2. LEHOUX, 1966-1968; AUTRAND, 2000.

3. REY, 1965, p. 494. 
Une grande partie de ces constructions est aujourd'hui disparue. Seuls subsistent les palais de Poitiers (actuel palais de Justice de la ville), de Bourges (Conseil général du Cher) et la Sainte-Chapelle de Riom (incluse dans le palais de Justice de la ville). On conserve en outre les ruines de plusieurs ensembles castraux dont seules celles de Mehun-sur-Yèvre montrent encore des parties en élévation.

La maîtrise d'ouvrage de Jean de Berry est un sujet largement débattu, mais un champ d'investigation reste encore ouvert aux chercheurs : celui des sources comptables. Les archives comptables de Jean de Berry forment un riche matériau qui permet d'éclairer, sous un jour nouveau, notre connaissance de la maîtrise d'ouvrage du prince et de l'organisation de ses chantiers. Dans un premier temps, cet article propose de faire une présentation de la gestion des œuvres de Jean de Berry d'après les sources comptables conservées. Cependant, il faut rappeler que les archives comptables ont été considérablement réduites depuis le $\mathrm{XV}^{\mathrm{e}}$ siècle et qu'elles se trouvent aujourd'hui dispersées entre différentes collections publiques à Paris et en régions. Ainsi, comme toute source historique, elles doivent être soumises à la critique avant d'être exploitées. C'est ce questionnement méthodologique qui occupe la seconde partie du présent article.

\section{Maîtrise d'ouvrage de Jean de Berry}

Depuis la fin du XIX ${ }^{\mathrm{e}}$ siècle, seules les constructions les plus prestigieuses de Jean de Berry ont fait l'objet de plusieurs publications ${ }^{4}$. Cependant, pour une bonne compréhension des sources, il est indispensable de poser d'abord la question de la maîtrise d'ouvrage du prince dans son acception la plus large prenant en compte les grands programmes comme les chantiers courants.

\section{Les entreprises importantes}

Il est clair que certains chantiers ont requis, autant par l'importance des moyens que par la portée politique des programmes, l'implication personnelle de Jean de Berry. Encore aujourd'hui, les vestiges archéologiques et un grand nombre de documents comptables témoignent de l'ampleur de ces entreprises représentatives des ambitions du prince. Cependant, l'inégalité de la documentation ne nous permet pas de décrire l'ensemble de ces entreprises et nous conduit à proposer deux catégories.

Une première catégorie concerne les constructions pour lesquelles nous possédons encore suffisamment de documents ou de vestiges archéologiques pour permettre d'en décrire l'histoire, le programme, les intervenants, etc. Les principaux châteaux et palais entrepris dans les trois dernières décennies du XIV siècle font partie des constructions bien documentées. Ils sont la manifestation de l'autorité

4. Champeaux et Gauchery, 1894 ; Magne, 1904; ChanCel-Bardelot et RaynaUd, 2004 ; Raynaud, 2004. 
princière sur ses terres. Parallèlement aux aménagements de confort (bibliothèques, étuves, latrines), Jean de Berry dote ses résidences de véritables symboles politiques dont les expressions les plus connues sont les Saintes-Chapelles et les «grandes salles", véritables nefs laïques.

À côté de cette catégorie, il existe d'autres constructions tout aussi importantes pour le prince mais qui ont depuis disparu et pour lesquelles la documentation fait défaut. Il est indispensable de rappeler ici l'existence de ces chantiers oubliés au premier rang desquels nous trouvons ceux de Paris et de la région parisienne (hôtel de Nesle, résidences de la Grange aux Merciers et de Bicêtre). Par ailleurs, Jean de Berry entretenait aussi dans son apanage des résidences de plaisance, des relais de chasse comme à Lusignan (Vienne), à Usson (Puy-de-Dôme) ou à Genouilly (Cher). Jean de Berry avait également des liens avec les communautés urbaines, avec plusieurs degrés de collaboration, en matière de construction, allant de l'aide financière à la prise en charge complète de la maîtrise d'œuvre. L'ouvrage qui tint le plus à cœur Jean de Berry fut sans aucun doute le «Gros Horloge» de Poitiers (beffroi installé en face de Notre-Dame-la-Grande). Enfin, faut-il encore évoquer les relations que Jean de Berry entretenait avec les communautés religieuses, notamment avec le chapitre Saint-Etienne de Bourges qui fit construire le «grand Housteau » par le général maitre des œuvres du prince, s'il faut en croire les anciens érudits ${ }^{5}$.

\section{Les chantiers courants}

L'activité du prince ne s'arrête pas aux grandes entreprises: une importante partie concerne l'entretien des équipements du domaine afin d'en maintenir les revenus ainsi que les moyens défensifs. Ces charges sont inhérentes à la fonction de prince apanagé. Dans la plupart des cas, l'implication du prince n'est pas nécessaire, les chantiers étant directement menés par les administrations locales. Cet aspect de la maitrise d'ouvrage de Jean de Berry n'est pas anodin car il représente souvent un poste de dépenses important pour les administrations locales et pèse lourd dans les finances ducales. Il ne s'agit pas d'ailleurs d'une particularité propre à Jean de Berry, l'étude des finances de Philippe le Hardi conduisant aux mêmes constatations ${ }^{6}$. Certains travaux de fortification ou d'aménagement urbain ont tout de même nécessité l'intervention du prince: Jean de Berry ne refuse pas l'aide financière que lui réclament les habitants de Poitiers pour restaurer leurs murailles ${ }^{7}$, il répond tout aussi favorablement à la demande des habitants de Niort qui lui réclament l'autorisation de faire aménager un port dans leur ville et confirme régulièrement leur droit de barrage et d'octroi ${ }^{8}$. Dans les premières années du $\mathrm{XV}^{\mathrm{e}}$ siècle, il nomme même, afin

5. CHAMPEAUX et GAUCHERY, 1894, p. 62. Cette attribution n'a jamais pu être véritablement prouvée.

6. JAQUIER, 2001, p. 154 ; RAUZIER, Jean, Finances et gestion d'une principauté au XIVe siècle, le duché de Bourgogne de Philippe le Hardi (1364-1384), Paris, Comité pour l'histoire économique et financière de la France, 1996, XI-766 p.

7. Provost, Catherine, Les travaux communaux dans la ville de Poitiers à la fin du XIVe siècle, mémoire de maîtrise d'histoire, Université de Poitiers, 2003, 2 vol.

8. LEHOUX, 1966-1968, t. I, p. 394-395, 419, 422-423; t. II, p. 262. 
certainement d'accélérer les travaux, Jean Chardon, lieutenant de son trésorier général, comme payeur des œuvres de cette entreprise.

\section{L'organisation des chantiers (encadrement technique et financier)}

Chaque chantier suppose au minimum la présence de fonds nécessaires, d'un maittre d'œuvre, d'une main d'œuvre qualifiée ainsi que des manœuvres. L'encadrement technique utilise invariablement deux systèmes fréquents sur les chantiers médiévaux : la régie et le marché. La régie impose au maittre d'ouvrage de mettre en place un encadrement technique assuré par un maître d'oeuvre pendant la durée de l'ouvrage ainsi que la rémunération à la «journée» des équipes d'ouvriers. Ce système permet un bon suivi du chantier, mais il est coûteux pour le maitre d'ouvrage qui, outre la surveillance, doit approvisionner le chantier en matériaux. Dans la mesure du possible, il préfère recourir au marché, c'est-à-dire établir un contrat d'adjudication au rabais et ne payer l'adjudicataire qu'à la réception des travaux. La direction des chantiers relève le plus souvent de procédures pragmatiques, mais on constate, pour les plus grands chantiers, l'émergence d'une administration spécifique.

\section{Une administration spécifique}

À partir du règne de saint Louis, on constate l'émergence de maçons institués par le roi et chargés d'assurer le suivi des œuvres royaux' ${ }^{9}$. Dès le milieu du XIVe siècle, le besoin urgent de fortification conduit à la mise en place d'administrations urbaines compétentes pour diriger les grands ouvrages ${ }^{10}$. À la fin du XIVe siècle, la présence, au sein des cours royales et princières, d'une administration chargée des œuvres est un phénomène courant. Dans son apanage, Jean de Berry reproduit le modèle de maitrise des œuvres royaux: un général maître des œuvres coordonnant l'action de lieutenants, un suivi financier assuré par des payeurs des œuvres, enfin des visiteurs des œuvres supervisant l'ensemble. Cette administration doit être capable de répondre à plusieurs impératifs. Elle doit assurer une réponse technique aux programmes et superviser toutes les étapes de la maitrise d'œuvre: expertises, devis, constitution des équipes, adjudication des marchés, suivi, acheminement des matériaux, réception des travaux, certification. Elle doit aussi justifier, dans une comptabilité spécialisée présentée devant la Chambre des comptes, l'utilisation des fonds alloués.

Le personnage central de cette administration est le général maitre des œuvres ${ }^{11}$. Le duc de Berry confie cette tâche à Guy de Dampmartin (1369-1397) puis à son frère Drouet de Dampmartin (1397-1413). Probablement d'origine parisienne, les frères Dampmartin travaillent dès 1365 au chantier royal de la grande vis du Louvre. Ils entrent peu de temps après au service de Jean de Berry. Entre 1367 et 1369,

9. CHAPELOT, 2001, p. 339-403.

10. RIgAUDIÈRE, Albert, «Le financement des fortifications urbaines en France du milieu du XIV e siècle à la fin du XVe siècle», Revue Historique, t. 553, janvier-mars 1985, p. 19-95.

11. ChampeauX et GaUChery, 1894, p. 75-86. 


\begin{tabular}{|c|c|c|c|}
\hline Fonction & Duché de Berry & Comté de Poitou & Duché d'Auvergne \\
\hline $\begin{array}{l}\text { Général maître } \\
\text { d'œuvre }\end{array}$ & \multicolumn{3}{|c|}{$\begin{array}{l}\text { Guy de Dampmartin }(1369-\dagger 1397 ?) \\
\text { Drouet de Dampmartin }(1398-\dagger 1413)\end{array}$} \\
\hline \multirow{3}{*}{$\begin{array}{l}\text { Lieutenants du géné- } \\
\text { ral maître d'œuvre }\end{array}$} & \multirow{3}{*}{\multicolumn{2}{|c|}{$\begin{array}{l}\text { Jean Guérart } \\
(1382-1416)\end{array}$}} & Pierre Juglard (1384) \\
\hline & & & Hugues Jouly (1384-1386) \\
\hline & & & Hugues Fouchier (1386-1410) \\
\hline
\end{tabular}

Tableau 1 : Les maîtres des œuvres de maçonnerie de Jean de Berry (1369-1416).

Guy prend les fonctions de général maître des œuvres et Drouet occupe plusieurs fonctions dont celle de concierge de l'hôtel du duc à Paris. À partir de 1380, Guy de Dampmartin réside à Bourges puis quitte cette ville pour s'installer huit ans plus tard à Poitiers. C'est dans ces deux villes qu'il reçoit les messagers de ses lieutenants et d'elles qu'il dirige les travaux de l'apanage. Drouet de Dampmartin, quant à lui, entre au service de Philippe le Hardi en qualité de général maître des œuvres. En 1398, il vient remplacer son frère, certainement décédé, auprès de Jean de Berry. Il s’installe durablement à Jargeau près d'Orléans, mais continue à exercer ses fonctions jusqu'à sa mort en 1413.

En l'absence du général maître des œuvres, les chantiers sont dirigés par un lieutenant. Certains d'entre eux, comme Hugues Fouchier en Auvergne ou Jean Guérart en Poitou et en Berry, connaissent d'importantes carrières après la mort du prince (tableau 1).

Les payeurs des œuvres ne sont pas des hommes de métier, mais des comptables chargés de la gestion des chantiers. Ils coordonnent les besoins des maîtres d'œuvre avec les fonds dont disposent les receveurs. Responsables financiers des chantiers, ils doivent consigner leur gestion dans des comptes dans lesquels est recopié d'une manière ordonnée le contenu de toutes les pièces comptables produites sur le chantier.

L'office de visiteur des œuvres est visiblement réservé à des officiers de haut rang. Charles V avait confié cette tâche à l'un de ses proches, Philippe Ogier qui, du reste, était chargé de la gestion financière des œuvres royaux ${ }^{12}$. Jean de Berry nomme également Arnoul Belin, le plus haut dignitaire de la Sainte-Chapelle de Bourges, nous ne connaissons malheureusement pas l'ampleur des prérogatives qui lui sont confiées ${ }^{13}$.

L'existence dans l'apanage d'une telle administration ne doit pas laisser penser qu'elle prenait en charge l'ensemble de la maîtrise d'ouvrage du prince. En dehors des grands chantiers, d'autres modalités d'encadrement sont utilisées.

\section{Autres acteurs et autres modalités}

Dans trois cas, il n'est pas fait mention d'une intervention de l'administration des œuvres de Jean de Berry: les chantiers parisiens, l'entretien du domaine, la rémunération des artistes.

12. Chapelot, 2001, p. 366-368.

13. BnF, ms fr. 20686 , fol. $58 \mathrm{v}$. 
Les chantiers parisiens, dont le plus important est l'hôtel de Nesle, ont certainement été pris en charge par l'administration royale. Quatre quittances nous apprennent que le payeur des œuvres royaux dans la prévôté de Paris, Jean Amyot, finançait le chantier de $\mathrm{Nesle}^{14}$. Nous savons par ailleurs que les princes du sang comme Louis d'Orléans faisaient appel à Raymond Du Temple, général maître des œuvres du roi, pour les travaux dans leurs résidences parisiennes ${ }^{15}$. Dans le cas de Berry, nous en sommes réduits aux hypothèses, faute de documents, mais il semble que les Dampmartin n'aient jamais dirigé un chantier parisien pour ce prince.

L'entretien du domaine relève des attributions de la Chambre des comptes ${ }^{16}$. Ses agents contrôlent régulièrement l'état des équipements : étangs, moulins, granges, résidences, etc. Les travaux d'entretien ou de réparation sont ensuite confiés aux administrations locales. Les dépenses apparaissent dans les chapitres « œuvres» de la comptabilité des châtelains ou des receveurs domaniaux ${ }^{17}$. Dans ce cas, le bâti n'est donc pas comptabilisé à part, il s'intègre complètement dans le mode de gestion du domaine. D'ailleurs, une partie des travaux est rémunérée en nature. Les travaux nécessitent un nombre restreint d'ouvriers, très souvent des artisans résidant à proximité des secteurs concernés. On note l'absence de payeur, les sommes sont parfois avancées par un riche fermier. L'entretien du domaine se fait donc selon des procédures ordinaires. Cependant si des travaux importants sont prévus, c'est-à-dire à l'initiative du duc, un maître d'œuvre est dépêché sur place. Dans ce cas, une comptabilité spécialisée (séparée des chapitres « œuvres » et rédigée à part) est produite ${ }^{18}$.

Enfin, le salaire d'un artiste, comme Jean de Cambray ou d'un André Beauneveu, n’apparaît jamais dans la comptabilité du général maître des œuvres. Les noms, les salaires ou les gages de ces artistes peuvent se rencontrer dans les comptes des mâ̂tres de la Chambre aux deniers ${ }^{19}$ et dans les comptes des receveurs des aides ${ }^{20}$. Dans certains cas, les sources sont pour le moins inattendues : en 1371, le paiement des artistes travaillant dans la chapelle ducale de la cathédrale de Bourges est inscrit dans un rôle d'imposition du Limousin ${ }^{21}$. La raison du mode particulier de rémunération des artistes tient au fait que Jean de Berry entretient avec eux des relations privilégiées et les pensionne au même titre que ses valets de chambre ${ }^{22}$. Le duc et ses proches conseillers tiennent probablement de cette manière à garder la haute main sur les programmes iconographiques et entendent traiter directement avec les sculpteurs

14. BnF, coll. Clairambault, vol. 4 , pièces n ${ }^{\circ} 85$ et 86 ; BnF PO 55, pièces n ${ }^{\circ} 11$ et 12 ; voir CHAPELOT, 2001, p. 363

15. Taveau-Launay, 2001, p. 323-338.

16. SCHNERB, Bertrand, «L'activité de la Chambre des comptes de Dijon entre 1386 et 1404, d'après le premier registre de ses mémoriaux ", in La France des principautés; les Chambres des comptes, $X I V^{e}$ et $X V^{e}$ siècle, Philippe Contamine et Olivier MATTÉONI (dir.), Comité pour l'histoire économique et financière, Paris, 1996, p. 55-64.

17. Bourges, Arch. dép. Cher, 8 G 2119-2132 (comptes de la baronnie de Graçay, 1372-1416).

18. Bourges, Arch. dép. Cher, 8 G 2122 et 2129 (chantier de l'hôtel de Genouilly).

19. Arch. nat., KK 250, fol. 34v ; KK 251, fol. 77 ; KK 252, fol. 66v.

20. BnF, PO 2580, 133-136.

21. BnF, ms fr. 23902 , fol. $2 \mathrm{v}-4 \mathrm{v}$

22. Arch. nat., KK 251, fol. 77. 
ou les peintres. Le duc rémunère donc les artistes indépendamment du calendrier et des modes de financement des chantiers. Les sources comptables illustrent ici une forme de mécénat ou plutôt un mode de protection dont bénéficient certains artistes au sein des cours royales ou princières.

Ainsi, l'encadrement des chantiers revêt un caractère très polymorphe et le pragmatisme, qui apparaît dans ces exemples, met en échec toute tentative d'établir une logique trop procédurale. Si la multiplicité des encadrements de chantier vient considérablement compliquer la recherche, sa mise en évidence permet aussi d'expliquer en grande partie la dispersion des pièces intéressant la construction.

\section{État des sources comptables}

Les publications consacrées aux constructions de Berry ont exploité à plusieurs reprises les sources comptables qui n'ont cependant encore jamais été présentées dans leur globalité. En outre, mise à part l'étude du registre de Barthélemi de Noce par Teilhard de Chardin ${ }^{23}$, il manque, dans les éditions anciennes, des indications diplomatiques nous permettant de connaître la nature des documents exploités (comptes, pièce originale ou note d'érudit) ou leur origine (comptabilité spécialisée ou non).

Avant d'entamer l'étude de ces sources, il est indispensable de rappeler que la production d'une comptabilité de chantier se fait en deux étapes. Une première phase se déroule en cours de chantier: le maître d'œuvre produit un certain nombre de documents qui certifient l'avancement des travaux et qui déclenchent le paiement par le payeur des œuvres. Ces documents de première main, rédigés sur le chantier par les hommes de métier, sont les plus intéressants. Parmi ces derniers, les devis et les contrats d'adjudication sont de loin les pièces les plus complètes car destinées à caractériser l'ouvrage à réaliser. Le maître d'œuvre tient également un journal hebdomadaire dans lequel il note le nombre de jours ouvrés et le salaire de chaque ouvrier. Dans un deuxième temps, parfois au bout de plusieurs années, le payeur des œuvres réunit dans un compte la teneur de ces différentes pièces et établit le bilan financier du chantier entre dépenses et recettes. À l'inverse des documents produits par le maître d'œuvre, la transcription des pièces comptables dans les comptes des payeurs des ouvres est parfois laconique.

Par nature, ces sources n'ont pas pour rôle d'établir des mémoires techniques mais d'apporter des garanties de bonne gestion. Pour cette raison, elles sont souvent peu éclairantes sur les choix artistiques, stylistiques ou iconographiques du commanditaire. En revanche, elles permettent de saisir d'une manière très large la gestion et l'organisation des chantiers.

Afin d'aborder l'analyse critique des sources comptables (quatrième partie), il est nécessaire de caractériser l'ensemble de cette documentation. Cette présentation est

23. Teilhard de Chardin, Emmanuel, «Registre de Barthélemi de Noces, officier du duc de Berry (1374-1377)», BEC, t. 52, 1891, p. 220-258 et 517-572. 
exposée en quatre parties consacrées à l'origine des sources, la méthodologie de recherche, la typologie des sources et la dispersion des sources.

\section{Origine des sources}

Si la documentation comptable est le reflet de l'organisation des chantiers, elle est avant tout celui des circuits de financement. À partir du milieu du XIV ${ }^{\mathrm{e}}$ siècle, devant l'insuffisance des revenus ordinaires liés aux besoins urgents de défense, les moyens de financer les bâtiments ont connu de grandes modifications ${ }^{24}$. Les villes, principaux maîtres d'ouvrage en matière de fortification, mettent en place de nouveaux circuits de financement en grande partie assis sur la fiscalité extraordinaire. Le produit de ces nouveaux impôts, prévus tout d'abord pour le paiement des troupes et des rançons, est la propriété exclusive du roi de France. Le recours à cette manne financière a donc pour contrepartie un droit de regard accru de l'autorité royale sur la gestion municipale. Les villes ne sont pas les seules à profiter de ce nouvel impôt: comme pour toutes les administrations princières, les grandes entreprises menées par Jean de Berry sont presque entièrement tributaires des subsides royaux. Cependant, ces nouvelles ressources n'ont pas remplacé les anciennes. On peut déterminer qu'un chantier important comme un château ou un palais pouvait être alimenté par plusieurs caisses. Le tableau 2 témoigne de la variété des circuits

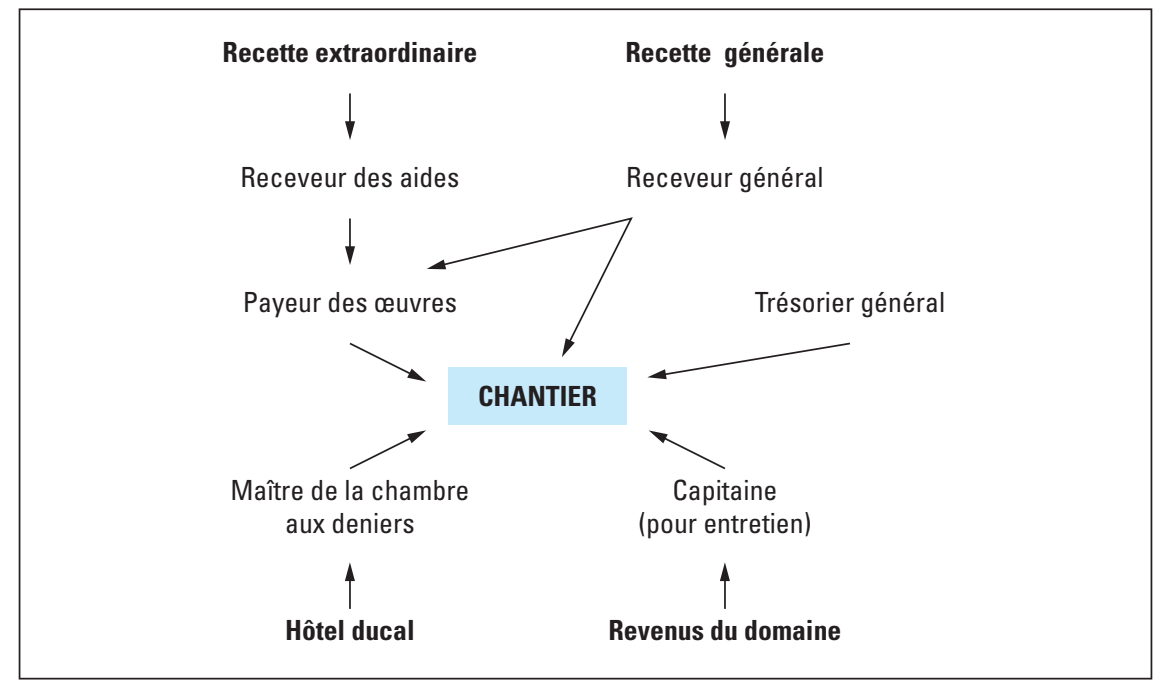

Tableau 2: Les financements des chantiers dans l'apanage de Jean de Berry recensés dans l'ensemble des archives comptables (1369-1409).

Ce schéma type est basé sur un recensement de tous les financements de chantiers inscrits dans les comptes. Il ressort des archives comptables de Jean de Berry qu'un chantier important (palais ou château par exemple) peut bénéficier d'un grand nombre de moyens de financement et que le payeur des ouvres ne gère pas l'ensemble des ressources.

24. RIGAUDIÈRE, «Le financement... », p. 19-95. 
permettant de financer un grand chantier. Il explique également le nombre important d'officiers pouvant intervenir, qu'ils appartiennent ou non à l'administration des œuvres du duc.

On peut dès lors opérer une séparation des sources comptables en deux ensembles: la comptabilité spécialisée et la comptabilité non spécialisée. La comptabilité spécialisée ne concerne que la construction. Elle est rédigée par les payeurs des œuvres pour les chantiers gérés par le général maître des œuvres du prince. Cette comptabilité est la mieux connue et la plus facile à inventorier. La comptabilité non spécialisée concerne l'ensemble des recettes et des dépenses courantes qui, de manière ponctuelle, peuvent contenir elles aussi des informations relatives à la construction. Il peut s'agir des comptes du maître de la Chambre aux deniers, des trésoriers généraux, des receveurs des aides ou des fouages, des agents de gestion domaniale. Contrairement à la comptabilité spécialisée, ce type de sources est très difficile à dépouiller car il se confond avec l'ensemble des archives comptables de l'administration du prince.

\section{Méthodologie et état actuel de la recherche}

Les grandes monographies consacrées au mécénat de Jean de Berry à la fin du $\mathrm{XIX}^{\mathrm{e}}$ siècle et au début du $\mathrm{XX}^{\mathrm{e}}$ siècle restent toujours la référence en matière d'archivistique bien que les sources exploitées procèdent davantage du sondage que de la recherche systématique. Le célèbre ouvrage écrit par Alfred de Champeaux et Paul Gauchery, Les travaux d'art exécutés pour Jean de France, duc de Berry, avec une étude biographique des artistes employés par ce prince s'appuie sur un très riche corpus de sources dont certaines sont malheureusement invérifiables. Les papiers de Paul Gauchery révèlent que les sources exploitées dans son ouvrage lui ont été communiquées par un grand nombre d'érudits avec lesquels il entretenait une correspondance nourrie ${ }^{25}$. La variété de ces sources explique qu'une partie des informations publiées est mal référencée. Du reste, l'usage du livre est compliqué par sa division en deux parties: la première partie (p. 1 à 46), qui reprend le contenu d'un article publié auparavant dans la Gazette Archéologique ${ }^{26}$, s'appuie principalement sur les comptes des Archives nationales. La rédaction de la deuxième partie (p. 49 à 72) a été motivée par le travail de Gabriel Ledos qui préparait une thèse de l'École des chartes sur la jeunesse du duc de Berry et qui mit au jour les manuscrits de la BnF. Le lecteur doit ainsi s'assurer que les hypothèses de la première partie se vérifient dans la deuxième. Heureusement, dix ans plus tard, Lucien Magne publie un ouvrage de synthèse ${ }^{27}$ beaucoup plus rigoureux et qui met en outre à la disposition du lecteur de longs extraits des sources comptables malheureusement mal édités et dépourvus d'apparat critique ${ }^{28}$.

25. Bourges, Arch. dép. Cher, 36 J 28-29.

26. Gazette Archéologique, 1887, p. 18-28, 64-71; 1888, p. 245-254.

27. MAGNe, 1904

28. FaVreau, Robert, La ville de Poitiers à la fin du Moyen Âge, Poitiers, SAO, 1978, t. I, p. XIII. 
En 1920, Léon Mirot, après un dépouillement incomplet des fonds des Nouvelles acquisitions françaises, des Quittances et pièces diverses et des Pièces Originales de la $\mathrm{BnF}$, a fait paraître un inventaire de pièces intéressant l'histoire de la construction sous le règne de Charles VI ${ }^{29}$. Ce premier dépouillement a permis la découverte de deux fragments de comptes relatifs au chantier de Riom autorisant une nouvelle datation de la Sainte-Chapelle ${ }^{30}$. Cet inventaire permet également de saisir la grande inégalité de répartition spatiale des pièces conservées dans ces trois collections: sur 860 pièces analysées, plus de 700 concernent la Normandie et le Vexin, alors que 14 seulement ont été produites par les chantiers de Jean de Berry (Riom ${ }^{31}$, Nonette ${ }^{32}$ et Paris). Aujourd'hui, l'étude globale de la maîtrise d'ouvrage de Jean de Berry doit nous conduire à pousser plus loin ces investigations pour deux raisons. Premièrement, la période retenue par Léon Mirot ne prend en compte que le règne de Charles VI (1380-1422) laissant dans l'ombre la décennie 1370 durant laquelle une partie des chantiers de Jean de Berry fut initiée. Enfin, certains documents découverts plus récemment, comme le manuscrit 7378 des Nouvelles acquisitions françaises (hôtel de Nesle), ont visiblement échappé au dépouillement de Léon Mirot. De même, Françoise Lehoux a entrepris le dépouillement des archives de Jean de Berry dans le cadre d'une étude historique. Son ouvrage en quatre volumes est précédé d'un inventaire des sources consultées tant à Paris que dans les collections publiques de province ainsi qu'à l'étranger. Même si cet inventaire ne permet pas de déterminer précisément toutes les pièces intéressant la construction, il n'en constitue pas moins la plus importante contribution à la présente recherche. Enfin, trois travaux universitaires concernant la Sainte-Chapelle de Bourges, les chantiers auvergnats ainsi que la production de carreaux de faïence pour le prince en Poitou et en Berry, ont donné lieu à l'édition de sources en partie inédites ${ }^{33}$.

Afin de compléter la liste des documents déjà édités, une approche méthodique de la question s'impose. La compréhension des cadres administratifs et financiers des chantiers autorise un dépouillement systématique des inventaires des fonds conservés aux archives nationales, départementales et communales susceptibles de contenir des comptes des payeurs des œuvres, des maîtres de la Chambre aux deniers, des trésoriers ou des receveurs. Précisons cependant que pour certains fonds auvergnats nous ne disposons encore que d'inventaires très sommaires. C'est le cas d'une partie du fonds Tiers-État de Basse Auvergne ${ }^{34}$ qui contient pourtant plusieurs registres intéressants; c'est le cas également du fonds de la Sainte-Chapelle de Riom comportant vingt cartons ${ }^{35}$.

29. Mirot, Léon, «Paiement et quittances de travaux exécutés sous le règne de Charles VI (13801422)», BEC, t. 81,1920 , p. 183-304.

30. BnF, ms fr. 26027 , fol. $2296-2297 \mathrm{v}$.

31. Dép. Puy-de-Dôme, chef-lieu de canton.

32. Dép. Puy-de-Dôme, cant. de Saint-Germain-Lembron.

33. SANCHEZ, 1992-1993; BON, 1992 ; RAYNAUD, 1996.

34. Clermont-Ferrand, Arch. dép. Puy-de-Dôme, 5 C 175-190.

35. Clermont-Ferrand, Arch. dép. Puy-de-Dôme, 27 G 1-20. 
En revanche ce mode de recherche n'est plus pertinent pour consulter les collections de la BnF car les pièces qu'elles contiennent ont perdu tout rapport avec leur classement d'origine. Les séries généalogiques, les collections Clairambault et Joursanvault sont organisées par ordre onomastique. Il faut alors procéder par recherche prosopographique, c'est-à-dire établir d'abord la liste nominative des officiers qui furent dans l'entourage du prince et qui auraient été susceptibles d'intervenir dans la construction (receveurs des aides, receveurs domaniaux, lieutenant du trésorier général, garde du sceau aux contrats, etc.). Les études historiques sur l'hôtel de Jean de Berry mettent en évidence 135 personnages liés à la construction ou appartenant aux services pouvant prendre en charge des chantiers de construction ${ }^{36}$. Il faut alors recouper ces noms avec les inventaires alphabétiques notamment celui de la très grande collection des Pièces Originales reconstituée à la fin du XIX siècle (120 volumes ont été consultés dans cette seule série). Le classement onomastique est peu commode : il impose une recherche systématique, peu efficace, forcément longue et fastidieuse. Trois dossiers contiennent des pièces très intéressantes et en partie inédites ${ }^{37}$ : Jean Chardon, lieutenant du trésorier général ${ }^{38}$, Martin Leroy, maître de l'hôtel du prince ${ }^{39}$, Jean Bésuchet, contrôleur de la dépense de l'hôtel ${ }^{40}$. Cependant, ce premier dépouillement des pièces originales reste incomplet car le nom indexé peut être simplement celui du tabellion qui a rédigé l'acte ${ }^{41}$. Heureusement, il est possible de procéder à un deuxième recoupement à partir des fiches d'inventaire chronologique. Cette vérification a permis de retrouver le dossier de Raymond Coustave, trésorier général du duc, dont l'orthographe du nom avait été mal recopiée ${ }^{42}$. Le dépouillement de la collection Clairambault, qui conserve son classement onomastique, d'après un inventaire chronologique (une trentaine de microfiches pour la période 1370-1416), a permis la mise au jour d'une quittance originale du payeur des œuvres du château d'Usson ${ }^{43}$ et d'un mandement inédit de Jean de Berry en faveur du chapitre Saint-Etienne afin que les chanoines puissent engager des travaux de couverture pour leur église sise dans son château de Gien ${ }^{44}$. Les dernières séries constituées à la fin du XIX ${ }^{\mathrm{e}}$ siècle, notamment celle des Quittances et pièces diverses, ont été conçues avec un classement chronologique. Le dépouillement de cette série a été peu fructueux car elle a déjà largement été exploitée dans le domaine de l'histoire de la construction.

36. LACOUR, René, Le gouvernement de l'apanage de Jean, duc de Berry, 1360-1416, Paris, Les presses modernes, 1934, p. VII-XXX; ToulgOët-TrÉANNA, «Les comptes de l'hôtel du duc (1370-1413)», Mémoires de la Société des Antiquaires du Centre, t. XVII, 1889-1890, p. 65-175.

37. Les pièces originales relatives à une famille ont été insérées dans des dossiers classés par ordre onomastique. Dans chacun des dossiers, les pièces sont collées sur des feuillets par ordre chronologique.

38. BnF, PO 678 .

39. BnF, PO 2580.

40. BnF, PO 324.

41. CROY, Joseph (Vicomte de), Notice historique sur les archives de la chambre des comptes de Blois, Blois, Grande Imprimerie de Blois, 1963, p. 496.

42. BnF, PO 868, dossier 19518 (Costane).

43. BnF, coll. Clairambault, vol. 142, pièce $\mathrm{n}^{\circ} 113$.

44. BnF, coll. Clairambault, vol. 218 , pièce $n^{\circ} 6$. 
Ces investigations dans les collections ont été complétées par la consultation des tables alphabétiques des manuscrits des fonds français et des Nouvelles acquisitions françaises ${ }^{45}$, réalisées à partir des notices des 17 volumes d'inventaires. Cet outil autorise une recherche rapide, mais ne permet pas de retrouver un élément non référencé dans les notices des catalogues. Pour pallier cet inconvénient, il est possible également de consulter les notices des inventaires et des catalogues des anciennes collections (Joursanvault, Bastard, par exemple). La consultation du catalogue de l'immense collection Joursanvault avant sa dispersion a permis de retrouver un mandement du duc de Berry ordonnant des réparations à l'étang de Mercurol en Auvergne $^{46}$. Trois volumes des Nouvelles acquisitions françaises et Nouvelles acquisitions latines provenant de la collection Bastard contiennent des extraits des registres capitulaires de la Sainte-Chapelle de Bourges (1402-1600) mentionnant plusieurs travaux entrepris après la mort du prince ${ }^{47}$. Enfin, les collections entrées récemment à la BnF sont les plus susceptibles de contenir des éléments inédits. C'est le cas de la collection réunie par Alexandre Laborde ${ }^{48}$, érudit passionné par l'histoire de l'art et des archives du Cogner (ancienne collection de Julien Chappée) dont le classement topographique facilite grandement la consultation ${ }^{49}$. Une dernière piste n'a pas encore été explorée: celle des fichiers de catalogues de vente d'autographes consultables à la $\mathrm{BnF}$ et qui, selon Michel Nortier, permettraient de retrouver un grand nombre de pièces comptables ${ }^{50}$.

\section{Typologie des éléments conservés : comptes et pièces justificatives}

Les comptes n'étaient jamais présentés seuls aux auditions de la Chambre des comptes: le comptable devait apporter également toutes les pièces justificatives des dépenses engagées qu'il avait utilisées pour rédiger son compte ${ }^{51}$. Le compte présente le grand avantage de permettre la lecture des dépenses dans la durée (une année généralement) et d'autoriser ainsi l'estimation du coût financier d'un chantier ainsi que sa progression. En revanche, le contenu des articles est souvent très succinct et

45. VIDIER, Alexandre et PERrier, Paul, Table générale alphabétique des Ancien et Nouveau fonds ( $\left.n^{\circ} 1-33264\right)$ et des Nouvelles acquisitions ( $n^{\circ} 1-10000$ ), Paris, E. Leroux, 1931-1948, 6 vol.

46. DE GAULLE, Catalogue analytique des archives de M. le Baron de Joursanvault contenant une précieuse collection de manuscrits, chartes et documents originaux au nombre de plus de quatre-vingt mille concernant l'histoire générale de France, l'histoire particulière des provinces, l'histoire de la noblesse et l'art héraldique avec un grand nombre de chartes anglo-françaises et de pièces historiques sur la Belgique, l'Italie et quelques autres états de l'Europe, Paris, Techener, 1838, 2 vol., lot n² 2681.

47. BnF, nouv. acq. fr., 1367-1368 et nouv. acq. lat., 1534. Mentionné par CHAMPEAUX et GAUCHERY, 1894, p. 87, mais sans aucune référence.

48. BnF, nouv. acq. fr., $22045, n^{\circ} 60$ : certificat d'Hugues Fouchier (1410), lieutenant de Drouet de Dampmartin en Auvergne; voir PERRIER, Paul, Chartes, lettres et pièces diverses relatives aux arts et aux artistes $d u X I V^{e}$ au XIXe siècle récemment donnés à la Bibliothèque nationale par M. le marquis et M. le comte Alexandre Laborde, Paris, Imprimerie nationale, 1915.

49. BnF, fonds Chappée, boîte LXXXI, $n^{\circ} 19$ : contrat d'adjudication pour le château et le palais de Poitiers, 1452.

50. NORTIER, 1965 , p. 486

51. JASSEMIN, Henri, La Chambre des comptes de Paris au XVe siècle, Paris, Auguste Picard, 1933, p. 112. 
apporte peu d'informations sur les travaux. Les pièces justificatives (journal du maître d'œuvre, marchés, quittances, certificats) représentaient un volume important: elles étaient reliées sommairement entre elles par ordre chronologique et étaient transportées dans des sacs ou dans des coffres. Certains de ces documents, comme les contrats d'adjudication de marché, sont des pièces de parchemin de très grand format qui contiennent beaucoup d'informations ${ }^{52}$. En théorie, comme le montre Michel Nortier, si les comptes manquent, les pièces justificatives doivent permettre d'en reconstituer le contenu ${ }^{53}$. Malheureusement, leur nombre a été considérablement réduit en raison de leurs mauvaises conditions de conservation: en vrac dans des sacs, des coffres ou des tonneaux.

\section{Dispersion des pièces}

Aujourd'hui, les pièces ou épaves de comptes produits par les chantiers de Jean de Berry font figure de rares rescapées. Même si il est très difficile d'estimer l'importance des documents perdus produits par la comptabilité spécialisée, on peut penser que le nombre total de comptes rédigés sur les chantiers de Jean de Berry devait approcher 150 environ (tableau 7). Or il ne subsiste qu'un compte complet et 14 épaves conservées aux Archives nationales et à la BnF (tableau 5). En outre, sachant qu'un compte spécialisé pouvait être accompagné de plus d'un millier de pièces justificatives ${ }^{54}$, on peut estimer le nombre de ces pièces produites à 150000 . On ne possède plus aujourd'hui que 17 pièces justificatives - mandements et quittances - conservées dans les différentes collections de la BnF. On peut donc estimer qu'il a été conservé un compte sur 10 et une pièce justificative sur 15000 .

La dispersion de la comptabilité non spécialisée a été encore plus importante: mis à part les comptes des maîtres de la Chambre aux deniers dans la série KK des Archives nationales, il ne reste presque plus rien des comptes de recette générale, des fouages ou des domaines.

Le tableau 3 montre que certaines des plus importantes résidences du prince, comme l'hôtel de Nesle, les châteaux de Bicêtre et de Mehun-sur-Yèvre, ne sont presque plus documentées. En revanche, ce n'est pas le cas d'édifices comme le château et le palais de Poitiers, le palais de Riom, l'hôtel de Genouilly et le château de Lusignan pour lesquels on conserve quelques comptes. Bien entendu, le type de source le plus riche est la comptabilité spécialisée, mais il convient néanmoins de ne pas négliger les autres, notamment celles pouvant nous informer sur l'emploi des artistes sur les chantiers.

Comme le montre le tableau 3, la dispersion des sources comptables de chantier n'est pas liée à l'importance des sites: c'est donc dans l'histoire des archives de Jean de Berry qu'il faut chercher une explication. L'installation de la Chambre des comptes de Bourges est consignée dans un document du 11 mai 1379 ${ }^{55}$. Cet acte

52. BnF, ms fr. 26 098, pièce $n^{\circ} 1892(35 \times 22$ cm) ; Clermont-Ferrand, Arch. dép. Puy-de-Dôme, 3 E PS $8(53,5 \times 37 \mathrm{~cm})$.

53. Nortier, 1965 , p. 460-537.

54. NORTIER, 1965, p. 467.

55. LeHOUX, 1966-1968, t. I, p. 436-437. 
affirmait la position centrale de Bourges au sein de l'apanage. L'absence d'héritier mâle à la mort du prince (1416) entraîna la rétrocession à la couronne de l'apanage et de ses archives, mais ce retour a certainement été différé par les événements dramatiques que connaissait le royaume de France à ce moment. L'institution reste en place à Bourges durant le début du règne de Charles VII. Le 6 novembre 1436, un édit royal ordonne l'installation de la Chambre des comptes à Paris. Le transfert des archives de la Chambre des comptes de Bourges vers Paris se fera trois ans plus tard ${ }^{56}$.

L'histoire de ce fonds n'a encore jamais été décrite, mais il est fort probable qu'il subit, comme celui de la Chambre des comptes de Blois ${ }^{57}$, d'importantes éliminations notamment au cours des déménagements ${ }^{58}$. L'accumulation de pièces justificatives a posé à l'évidence un grand problème de conservation car elles représentaient un volume considérable pour des locaux devenus rapidement trop exigus. À la différence des comptes, les pièces justificatives sont laissées en vrac, sans tri ni classement ${ }^{59}$. Parfois, elles sont trouvées à même le sol, puis ramassées sans égard. La conséquence de ce manque de soin est souvent l'élimination des pièces jugées inutilisables car trop "pourries ${ }^{60}$. Les archives de Jean de Berry subirent certainement le même sort. À leur retour à Paris, les archives de la Chambre des comptes de Bourges furent probablement entreposées dans diverses annexes de la Chambre des comptes regroupant les comptes et les pièces audités qui ne présentent pas un intérêt pour la gestion quotidienne de la Chambre ${ }^{61}$. Ces dépôts, situés dans le couvent des frères prêcheurs et dans la salle Barrin près de la Sainte-Chapelle, répondaient plus à un souci de stockage qu'à une véritable volonté de classement et de conservation. D'ailleurs, l'office de garde des livres chargé de la conservation n'apparait qu'en $1520^{62}$. C'est certainement le peu d'intérêt que ces archives offraient pour la Chambre des comptes qui favorisa leur pillage par les érudits, les collectionneurs et les généalogistes (Gaignière, Clairambault, d'Hozier) dès la seconde moitié du XVII siècle. Paradoxalement, l'acquisition par la Bibliothèque royale des pièces justificatives et des comptes rassemblés par ces personnages a permis leur sauvegarde alors que les documents restés sur place ont été détruits en grande majorité par la suite.

Le tableau 3 montre l'importance de la dispersion des informations relatives à la construction contenues dans les archives comptables de Jean de Berry. Cette dispersion est de surcroît très inégale: certains chantiers sont richement documentés (Poitiers, Riom, Lusignan) alors que d'autres conservent à peine quelques témoins de leur histoire. Le cas le plus exemplaire est celui de l'hôtel de Nesle à Paris, principale résidence du duc de Berry, mais dont la documentation comptable se limite aujourd'hui à quatre quittances.

56. Monestier, R., « Note sur une maison de Bourges, l'ancienne Chambre des Comptes du duc Jean de Berry ", Mémoires de la Société des Antiquaires du Centre, t. 40, 1921, p. 213.

57. Croy, Notice historique...

58. NORTIER, 1965, p. 465.

59. Ibidem, p. 468

60. CROY, Notice historique..., p. 117.

61. NORTIER, 1965, p. 461-470.

62. Ibid., p. 469. 


\begin{tabular}{|l|c|c|c|c|c|c|c|}
\hline \multicolumn{1}{|c|}{ Chantiers } & A & B & C & D & E & F & Totaux \\
\hline Hôtel de Nesle (Paris) & & 1 & & & & 4 & 5 \\
\hline $\begin{array}{l}\text { Hôtel de la rue de l'Echelle du Temple } \\
\text { (Paris) }\end{array}$ & & 3 & & & & & 3 \\
\hline Palais et château de Poitiers (Vienne) & 3212 & & & & & & 3212 \\
\hline Château de Lusignan (Vienne) & 218 & & & & & & 218 \\
\hline Palais de Bourges (Cher) & 130 & & 4 & & & & 134 \\
\hline Château de Mehun-sur-Yèvre (Cher) & 8 & 3 & 2 & & & & 13 \\
\hline $\begin{array}{l}\text { Chapelle de la cathédrale de Bourges } \\
\text { (Cher) }\end{array}$ & & 2 & 2 & & & & 4 \\
\hline Hôtel de Genouilly (Cher) & & & & & 444 & & 444 \\
\hline Palais de Riom (Puy-de-Dôme) & 688 & & 1 & 1 & & 8 & 698 \\
\hline Château de Nonette (Puy-de-Dôme) & & & & 2 & & 3 & 5 \\
\hline Château d'Usson (Puy-de-Dôme) & 92 & & & & & & 92 \\
\hline Domaine de Graçay (Cher) & & & & & 208 & & 208 \\
\hline Port de Niort (Deux-Sèvres) & & & & & & 2 & 2 \\
\hline Château de la Réole (Gironde) & & & 1 & & & & 1 \\
\hline $\begin{array}{l}\text { Rémunération de peintres et sculpteurs } \\
\text { à Bourges et à Poitiers }\end{array}$ & & 3 & 7 & & & & 10 \\
\hline Totaux & 4348 & 12 & 17 & 3 & 652 & 17 & 5049 \\
\hline & & & Nombre total d'article : 5049 \\
\hline
\end{tabular}

Tableau 3 : Les chantiers de Jean de Berry a dans les sources comptables (1369-1416). Répartition par site du nombre d'articles ${ }^{\mathrm{b}}$ relatifs à la construction.

A : comptes des payeurs des œuvres (comptabilité spécialisée), B : comptes des maîtres de la Chambre aux deniers, $\mathrm{C}$ : comptes des receveurs (généraux et fouages), D: comptes des trésoriers généraux, $\mathrm{E}$ : comptes des receveurs domaniaux, $\mathrm{F}$ : pièces justificatives (quittances et mandements).

a. Ce tableau recense uniquement les chantiers pour lesquels Jean de Berry est maitre d'ouvrage.

b. J'entends par «article» le nombre d'items contenus dans chaque compte et que l'on peut considérer comme une unité d'information. Par exemple, une pièce justificative vaut pour un «article».

En 1737, l'incendie de la Chambre des comptes de Paris ravagea le dépôt du garde des livres. Les décisions prises afin de réorganiser les fonds sauvés exigèrent l'élimination des pièces antérieures à $1640^{63}$. Cependant, quelques documents échappèrent à la destruction pour venir grossir les grandes collections de Gevigney, Beaumarchais et Joursanvault constituées avant la Révolution. C'est après ce moment que commence véritablement la dispersion des documents. Mises à part les collections Gevigney et Beaumarchais qui furent intégralement acquises par la Bibliothèque royale, les autres grandes collections des XVIII et $\mathrm{XIX}^{\mathrm{e}}$ siècles ne rejoignirent les collections nationales qu'après avoir été dispersées et vendues en lots. Même si

63. NORTIER, 1965 , p. 472. 
la Bibliothèque nationale, sous l'administration de Léopold Delisle, a pris conscience de l'importance de ces pièces et a entrepris de les racheter, on recense toujours une masse considérable de pièces justificatives émanant de la Chambre des comptes dans les collections privées, les archives départementales mais aussi à l'étranger ${ }^{64}$.

Certains documents intéressant la construction n'ont cependant pas suivi le retour des archives de Jean de Berry à Paris et demeurent toujours dans les services d'archives locaux. En premier lieu, des archives ont suivi des donations faites par Jean de Berry. Le fonds de la Sainte-Chapelle de Bourges (Archives départementales du Cher) conserve ainsi les comptes de la terre de Graçay qui avait été réunie à son temporel par le prince. Cette série est unique en son genre car il s'agit des seuls comptes domaniaux de Jean de Berry conservés ${ }^{65}$. Le fonds de la Sainte-Chapelle contient également un document insolite: un fragment de compte de payeur des œuvres qui servit de couverture à un registre du $\mathrm{XV}^{\mathrm{e}}$ siècle, ce qui montre au demeurant le peu d'importance que revêtait ce genre de document pour les archivistes du chapitre ${ }^{66}$. Par ailleurs, certains services d'archives possèdent également les doubles des comptes transportés et disparus à Paris. La raison en est simple: les comptables devaient présenter leurs registres en double afin de conserver un exemplaire avec eux après audition. Les Archives départementales du Puy-de-Dôme conservent ainsi plusieurs doubles de comptes du receveur des fouages ${ }^{67}$ et un cahier de papier contenant la comptabilité du trésorier général ${ }^{68}$. Enfin, les services d'archives municipaux ou départementaux ont cherché à récupérer dans les ventes publiques des pièces intéressant l'histoire régionale: en Auvergne, une association d'historiens fit l'acquisition d'un mandement du prince et le versa dans la série F des Archives départementales du Puy-de-Dôme ${ }^{69}$.

En dernier lieu, signalons que les archives concernant les travaux urbains dont Jean de Berry n'était pas le maître d'ouvrage, mais auxquels il apporta une aide financière ou logistique, sont restées la possession des communes. Les archives de Poitiers, par exemple possèdent plusieurs cartons nous permettant de suivre les travaux de fortification de la ville et la construction du Gros Horloge ${ }^{70}$. En Auvergne, un long contrat d'adjudication passé à Aigueperse pour un marché de maçonnerie impliquant un lieutenant de Drouet de Dampmartin était jusqu'à présent passé inaperçu. Ce contrat faisait partie du fonds d'Aigueperse déposé récemment aux Archives départementales du Puy-de-Dôme ${ }^{71}$. Il est très probable que d'autres documents de ce genre

64. NORTIER, 1965 , p. 487-537.

65. Bourges, Arch. dép. Cher, 8 G 2119-2132.

66. Bourges, Arch. dép. Cher, 8 G 1166 (compte du payeur des œuvres du palais de Bourges, 21 mai 138121 mai 1382). Ce compte a été édité par CravaYAT, Paul, «Fragment d'un compte de l'œuvre du Palais de Bourges, 1381-1382 », Mémoires de l’Union des Sociétés Savantes de Bourges, t. IV, 1955, p. 7-23.

67. Clermont-Ferrand, Arch. dép. Puy-de-Dôme, 5 C 184.

68. Clermont-Ferrand, Arch. dép. Puy-de-Dôme, 5 C 179.

69. Clermont-Ferrand, Arch. dép. Puy-de-Dôme, 1 F 186, pièce $n^{\circ} 1$.

70. Provost, op. cit.

71. Clermont-Ferrand, Arch. dép. Puy-de-Dôme, 3 E PS 8. Je tiens à remercier Marie-Astrid Zang, conservateur du patrimoine, d'avoir attiré mon attention sur ce document. 
restent encore à découvrir dans la masse de documents comptables (comptes ou pièces justificatives) conservés dans les régions qui furent gouvernées par Jean de Berry ${ }^{72}$.

\section{Analyse critique et reconstitution des sources comptables : l'exemple de la comptabilité spécialisée}

\section{Les comptes des payeurs des ouvres de Jean de Berry}

Les payeurs des œuvres de Jean de Berry ont laissé une documentation très inégale qui permet cependant d'apporter un éclairage précieux sur presque tous les grands programmes que le prince mena en Berry, en Poitou et en Auvergne. Notre connaissance du réseau des payeurs des œuvres installés par Jean de Berry (tableau 4) n'est pas complète. Par exemple, nous ne gardons que des mentions très sommaires prouvant l'activité de Robert Loyson, payeur des œuvres du palais de Riom ${ }^{73}$, et de Jean Chardon, payeur des œuvres du port de Niort.

\begin{tabular}{|c|c|c|c|}
\hline \multicolumn{2}{|c|}{ Payeur des œuvres } & Chantier & Dates d'exercice \\
\hline \multirow{3}{*}{ Poitou } & Étienne Gervais & $\begin{array}{l}\text { Château et palais } \\
\text { de Poitiers (Vienne) }\end{array}$ & 8 novembre $1382-28$ février 1388 \\
\hline & Jean Aubereau & $\begin{array}{l}\text { Château de Lusignan } \\
\text { (Vienne) }\end{array}$ & 26 novembre 1397 - 26 novembre 1403 \\
\hline & Jean Chardon & $\begin{array}{l}\text { Port de Niort } \\
\text { (Deux-Sèvres) }\end{array}$ & Mentionné en 1403 \\
\hline \multirow{3}{*}{ Auvergne } & Robert Loyson & $\begin{array}{l}\text { Palais de Riom } \\
\text { (Puy-de-Dôme) }\end{array}$ & Avant le $1^{\text {er }}$ août 1376 \\
\hline & Jean de Savignon & $\begin{array}{l}\text { Palais de Riom } \\
\text { et château } \\
\text { de Nonette } \\
\text { (Puy-de-Dôme) }\end{array}$ & $1^{\text {er }}$ août $1376-1^{\text {er }}$ août 1389 \\
\hline & Jean Bouton & $\begin{array}{l}\text { Château d'Usson } \\
\text { (Puy-de-Dôme) }\end{array}$ & 7 décembre 1388 - 24 mai 1389 \\
\hline \multirow[t]{2}{*}{ Berry } & $\begin{array}{l}\text { Huguenin } \\
\text { Meschin }\end{array}$ & $\begin{array}{l}\text { Palais de Bourges } \\
\text { et château de } \\
\text { Mehun-sur-Yèvre } \\
\text { (Cher) }\end{array}$ & 21 mai $1380-21$ mai 1382 \\
\hline & $\begin{array}{l}\text { Jean } \\
\text { de Chalemaigne }\end{array}$ & $\begin{array}{l}\text { Château } \\
\text { de Mehun-sur-Yèvre }\end{array}$ & $1^{\text {er }}$ octobre $1407-30$ septembre 1409 \\
\hline
\end{tabular}

Tableau 4 : Les payeurs des œuvres de Jean de Berry.

Les payeurs des œuvres rédigent leurs comptes selon des règles bien décrites par les spécialistes de la comptabilité médiévale. Les comptes des payeurs des œuvres

72. LeHOuX, 1966-1968, t. I, p. XVII-XXIII : Carcassonne, Arch. dép. Aude; Rodez, Arch. dép. Aveyron; Aurillac, Arch. dép. Cantal; Saint-Flour, Arch. mun.; Toulouse, Arch. mun.; Niort, Arch. dép. Deux-Sèvres; Montauban, Arch. dép. Tarn et Garonne.

73. BnF, ms fr. 11488 , fol. $1-v$. 
répondent tous au même double impératif: faire l'état ordonné de toutes les recettes et dépenses engagées sur le chantier au cours de l'année et apporter la preuve du bon emploi des deniers versés. La connaissance des procédures suivies par les payeurs et de la structure des comptes facilite grandement leur étude et permet de reconstituer les lacunes laissées par la disparition des feuillets.

Le plus souvent, la comptabilité de chantier est consignée en exercices annuels. Les termes de ces exercices sont très variables d'un chantier à l'autre. Étienne Gervais (château et palais de Poitiers) clôt ses exercices au mois de février, Huguenin Meschin (palais de Bourges) au mois de mai, Jean de Savignon (palais de Riom) au mois d'août, Jean de Chalemaigne (château de Mehun-sur-Yèvre) au mois d'octobre et Jean Bouton (château d'Usson) au mois de décembre. Les quantièmes, marquant le début de chaque compte, sont variables car tous les comptes d'œuvres de Jean de Berry commencent par un lundi. Seule exception: les comptes de Jean de Savignon à Riom sont clos tous les 31 juillet. Pour réaliser l'état de toutes les dépenses, le payeur doit collationner et synthétiser toutes les pièces justificatives dressées et certifiées en cours de chantier par le maittre d'ouvre (journal, contrats d'adjudication, quittances). C'est à partir de cette matière que le payeur retranscrit ses rubriques. Ces comptes ne sont donc pas réalisés en cours de chantier; ce sont des synthèses soignées de la comptabilité de chantier. Le procédé du payeur rejoint ici celui du maitre de la Chambre aux deniers rédigeant ses comptes à partir des rôles mensuels délivrés par l'hôtel princier $^{74}$. Pour apporter la preuve du bon emploi des sommes versées sur le chantier, le payeur des œuvres doit justifier chaque dépense par un acte authentifié. Une fois l'ensemble de ces pièces justificatives en sa possession, le payeur des œuvres commence l'écriture de son compte. Les exemples de comptes conservés montrent que la rédaction se fait sur des cahiers de huit feuillets reliés entre eux pour constituer un registre. Le tableau 6 montre que les registres les mieux conservés contenaient une dizaine de cahiers. Un registre peut également réunir plusieurs comptes (voir tableau 9, compte $\mathrm{n}^{\circ} 6$ : compte du payeur des œuvres du palais de Riom, $1^{\text {er }}$ août $1382-$ $1^{\text {er }}$ août 1384). Sur le premier feuillet, le payeur des œuvres présente le compte en indiquant son identité, le secteur géographique ou les monuments sur lesquels s'étendent ses compétences ainsi que les termes de l'exercice. À la suite de cette présentation, le payeur des œuvres insère une copie de sa lettre de commission. Cette copie n'est pas reportée sur chacun des comptes, mais uniquement sur le premier compte rendu par ce payeur ${ }^{75}$. Si le payeur des œuvres s'octroie les services d'un procureur pour rendre ses comptes, il prendra soin de retranscrire également la lettre de procuration $^{76}$.

Faisant suite aux recettes (présentées par origine et par ordre chronologique des versements), la partie consacrée aux dépenses est divisée en deux ensembles bien distincts: les travaux en régie et les marchés baillés à tâche. Le payeur doit

74. JASSEMIN, La Chambre des comptes..., p. 118.

75. BnF, ms fr. 20686 , fol. 13-v (premier compte d'Étienne Gervais, 9 février 1383-8 janvier 1384); BnF, ms fr. 11488 , fol. 1-v.(compte de Jean de Savignon, $1^{\text {er }}$ août $1382-1^{\text {er }}$ août 1384 ).

76. BnF, ms fr. 20 686, fol. 42 et 50 (compte de Jean Aubereau pour le château de Lusignan, 2 décembre 1399 - 26 novembre 1403$)$. 
impérativement les enregistrer séparément car la nature des pièces justificatives diffère selon le cas. Le système de régie implique la présence sur le chantier pour surveiller et diriger les équipes d'ouvriers du maître d'œuvre. Celui-ci tient une comptabilité journalière appelée « livre de contrôle» ou «papier» du maître d'œu$v{ }^{77}$. Ce document certifie le nombre de jours ouvrés et le salaire de chaque ouvrier; il déclenche le paiement des dits ouvriers par le payeur des œuvres. Ces rôles sont rendus avec la comptabilité du chantier au titre de pièces justificatives ${ }^{78}$. Dans son compte, le payeur des œuvres reproduit le rythme hebdomadaire et le contenu de ces rôles, on compte donc cinquante-deux rubriques hebdomadaires ou « journées d'ouvriers» (tableau 8). Les travaux en régie occupent la majeure partie du compte. Ces rubriques apportent une contribution très précieuse à la connaissance des chantiers car elles permettent de suivre les travaux en temps réel, mais aussi de connaître l'identité du personnel employé, l'organisation des équipes, le nombre de jours ouvrés, la quantité et la nature des matériaux acheminés à pied d'œuvre.

Dans le cas des marchés baillés à tâche, le maître d'œuvre, après avoir établi des devis, accorde par contrat d'adjudication les marchés aux entreprises offrant les conditions les plus intéressantes. Une fois les travaux terminés, il dresse un certificat établissant la conformité des travaux avec le devis et par ce certificat, il autorise le paiement par le payeur des œuvres. Enfin, l'entreprise émet une quittance en échange de sa rémunération. Les dépenses pour marchés, n'étant pas indexées sur le nombre de jours ouvrés, ni sur le nombre d'ouvriers, mais sur un montant global fixé à l'avance, doivent être insérées dans le compte séparément des parties consacrées aux travaux en régie. Dans les comptes d'œuvre de Jean de Berry, on les rencontre après les journées d'ouvriers (le nombre de marchés retranscrits dans un compte dépasse rarement la vingtaine). Contrairement aux travaux en régie, le grand intérêt de ces rubriques est la description des ouvrages dont les détails peuvent être très éclairants. Cependant, les données concernant les hommes, les journées travaillées voire les matériaux sont masquées derrière la figure unique de l'adjudicataire.

Sur les derniers feuillets, le payeur des œuvres mentionne les frais annexes. Parmi ceux-ci, on trouve les gages du personnel de gestion : le maitre d'œuvre, le payeur des œuvres, voire le cas échéant, les vacations du visiteur des œuvres ou du général maître des œuvres. Sur le dernier feuillet sont notés les frais d'écriture, très précieux pour connaître le nombre de feuillets que contenaient les comptes à l'origine.

77. Nous trouvons dans la commission de Huguenin Meschin, payeur des œuvres du palais de Bourges et du château de Mehun-sur-Yèvre en 1380 (BnF, ms fr. 20 686, fol. 8) « [...] Le livre de contrerolle a este rendu par le dit payeur avec cest present compte en la dite chambre». De même, dans les comptes de Jean de Savignon payeur des œuvres du palais de Riom, 1382-1384 (Arch. nat., KK 255, fol. 28v) : «Ung grant papier achapte pour escripre la mise et la despence qui ce feroit en chacune sempmayne pour maniere de contrerolle par ledit Pierre Juglar comme lieutenant dudit meistre Guy es euvres dudit palais ».

78. Aucun de ces documents n'a été conservé. Les hasards de la conservation produisent parfois le phénomène inverse: le chantier de l'Hôtel-Dieu de Bourges n'est documenté que par les pièces justificatives: cf. HAMON, Étienne, «Les débuts du chantier de l'Hôtel-Dieu de Bourges d'après les sources comptables ", BEC, 2003, t. 161, p. 9-32. 
L'exposé des différentes parties des comptes des payeurs des œuvres montre qu'ils étaient conçus sur une structure très classique commune à la plupart des registres comptables de l'époque.

Les comptes des payeurs des œuvres, comme l'ensemble des documents comptables, étaient destinés à être examinés par la Chambre des comptes. Les annotations marginales latines sur les feuillets nous permettent de suivre les étapes de réception, d'examen et de jugement ${ }^{79}$. L'étude de ces mentions fut souvent négligée par les historiens de Jean de Berry même si, dès le début du XX $\mathrm{XX}^{\mathrm{e}}$ siècle, Alfred de Gandilhon en souligne l'importance à propos des comptes de la baronnie de Graçay ${ }^{80}$. La vérification du compte se fait d'après les pièces justificatives: régularité des mandements princiers, conformité des contrats d'adjudications et des quittances. En outre, chaque pièce doit comporter les signatures requises. Le payeur doit rendre les pièces justificatives classées par ordre chronologique pour permettre à l'examinateur de les vérifier aisément en suivant l'ordre des rubriques: dans les comptes, on ne trouve pas de système d'indexation des actes et les rares pièces justificatives conservées ne portent pas de numérotation. Toute dépense signalée dans le compte, mais qui ne prouverait pas son authenticité dans un acte joint au compte fait l'objet d'une annotation de l'examinateur. Elle est définitivement rayée si le payeur n'apporte pas la pièce manquante. Les remarques et les corrections sont indiquées sous forme d'expressions normalisées par lesquels l'auditeur prépare la future correction du compte. Par exemple, il indique que la dépense a été vérifiée (videatur) ou que la recette a été versée par un receveur dont il faudra ressortir le compte (corrigatur). Les dépenses invalidées sont indiquées (radiatur) puis rayées purement et simplement du compte. Ces notes conduisent aussi les membres du bureau de la Chambre des comptes à questionner le payeur sur un paiement sans justificatif (loquatur). Par exemple, Huguenin Meschin, payeur des œuvres du palais de Bourges, doit s'expliquer sur un travail baillé à tâche, mais dont le marché n'avait pas été passé selon les procédures adéquates ${ }^{81}$.

C'est après la validation des pièces justificatives et la radiation des articles erronés que l'auditeur peut procéder au calcul des sommes afin de déterminer la balance du compte ${ }^{82}$. Pour éviter les erreurs, l'auditeur procède par étapes intermédiaires aussi appelées «fausses sommes». La fausse somme couramment employée est la somme hebdomadaire inscrite au terme de chaque semaine. Elle est précédée de la mention summa ou S. Selon les comptes, on rencontre aussi des sommes mensuelles, bimensuelles voire semestrielles. Une fois établies ces «fausses sommes», les auditeurs calculent les « grosses sommes », c'est-à-dire le total de chacune des grandes parties du compte: d'un côté le total des recettes (summa recepti), de l'autre la somme

79. Les comptes domaniaux de Graçay de 1409 à 1412 font exception à la règle : ils ont été audités en français durant cette période; Bourges, Arch. dép. Cher, 8 G 2128-2130.

80. GANDilhon, Alfred (de), «Les terres de Vatan et de Graçay, et Jean de France, duc de Berry (1370-1405) », Mémoires de la Société des Antiquaires du Centre, t. 30, 1906, p. 55-86.

81. Bourges, Arch. dép. Cher, $8 \mathrm{G}$ 1166, fol. 4v (comptes d'Huguenin Meschin, palais de Bourges, 21 mai 1381 - 21 mai 1382); Loquatur quod dictus mercatus non fuit traditus in sollemnitate qua decet. 82. JASSEMIN, 1933, p. 139. 
de «toute» des dépenses (summa expensi) qui comprend les paiements des travaux en régie (summa dietarum), les paiements des marchés baillés à tâche (summa tascharum) et les paiements des gages (summa vadiorum). Dans les comptes d'Étienne Gervais et de Jean de Savignon, on rencontre les mentions prima grossa et secunda grossa: il s'agit là de notions variables recouvrant parfois une année ${ }^{83}$, six ou sept mois ${ }^{84}$.

\section{Critique et reconstitution d'une documentation lacunaire}

Sur les 150 volumes probablement rendus par les payeurs des œuvres, il subsiste 287 feuillets provenant de quinze comptes différents qui concernent huit chantiers répartis dans l'ensemble de l'apanage du prince (Berry, Poitou, Auvergne) et sont de la main de six payeurs des œuvres. Sur ces quinze comptes, un seul est demeuré complet, les autres sont réduits à l'état d'épaves. La répartition de l'ensemble des 287 feuillets conservés (tableau 6) est la suivante: le palais et château de Poitiers (191 feuillets), le palais de Riom (61 feuillets), le château de Lusignan (20 feuillets), le château d'Usson (6 feuillets), le palais de Bourges et le château de Mehun-sur-Yèvre (7 feuillets). La dispersion des feuillets dans le temps et dans l'espace exige un travail de reconstitution des comptes et des feuillets manquants passant par un examen codicologique de la documentation conservée. Cette technique doit permettre de caractériser la production de chaque payeur des œuvres afin de redonner leur place, dans les comptes disparus, aux feuillets dispersés.

La reconstitution des comptes ainsi pratiquée met en évidence trois types de lacunes: la disparition de comptes au sein d'une série, la disparition de feuillets dans des comptes partiellement conservés, les procédés variables de transcription des payeurs des œuvres.

\begin{tabular}{|c|c|c|c|c|c|}
\hline \multicolumn{4}{|c|}{ Comptes } & \multicolumn{2}{|c|}{$\begin{array}{c}\text { Datation } \\
\text { des exercices }\end{array}$} \\
\hline $\mathrm{N}^{\circ}$ & Références/dépôt & Désignation & Payeur des œuvres & $\begin{array}{l}\text { Début } \\
\text { (ns) }\end{array}$ & Fin (ns) \\
\hline 1 & $\begin{array}{l}\text { BnF, ms fr. } 20686 \text {, } \\
\text { fol. } 13-20 v\end{array}$ & $\begin{array}{l}\text { Comptes du palais, } \\
\text { du château et du bateau } \\
\text { de Poitiers }\end{array}$ & Étienne Gervais & 09-fév-83 & 07-fév-84 \\
\hline 2 & $\begin{array}{l}\text { BnF, ms fr. } 20 \text { 686, } \\
\text { fol. 9-12v et Arch. nat., } \\
\text { KK } 256 \text {, fol. } 1-56 \mathrm{v}\end{array}$ & $\begin{array}{l}\text { Comptes du palais, } \\
\text { du château et du bateau } \\
\text { de Poitiers }\end{array}$ & Étienne Gervais & 08-fév-84 & 12-fév-85 \\
\hline 3 & $\begin{array}{l}\text { Arch. Nat., KK 257a, } \\
\text { fol. 1-39v }\end{array}$ & $\begin{array}{l}\text { Comptes du palais, } \\
\text { du château et du bateau } \\
\text { de Poitiers }\end{array}$ & Étienne Gervais & 13-fév-85 & 11-fév-86 \\
\hline
\end{tabular}

83. Arch. nat., KK 255, fol. 6v (Riom, $1^{\text {er }}$ août $1382-1^{\text {er }}$ août 1383).

84. Compte de Riom ( $1^{\text {er }}$ août $1382-1^{\text {er }}$ août 1384 ), Arch. nat., KK 255 , fol. $20 \mathrm{v}$ et 46 . La première grosse comprend les mois d'août jusqu'à janvier inclus; la deuxième du mois de février jusqu'au $1^{\text {er }}$ août; compte de Poitiers (Poitiers, 8 février 1384 - 12 février 1385), Arch. nat., KK 256, fol. $54 \mathrm{v}$ : Secunda grossa, summa mesum augusti, septembris, octobris, novembris, decembris, januarii et februarii $1973 \mathfrak{E} 3 \mathrm{~s} 10$ d. $t$. 


\begin{tabular}{|c|c|c|c|c|c|}
\hline 4 & $\begin{array}{l}\text { Arch. Nat., KK 257b, } \\
\text { fol. 1-85v }\end{array}$ & $\begin{array}{l}\text { Comptes du palais, } \\
\text { du château et du bateau } \\
\text { de Poitiers }\end{array}$ & Étienne Gervais & 12-fév-86 & 10-fév-87 \\
\hline 5 & $\begin{array}{l}\text { BnF, ms fr. } 20686 \\
\text { fol. } 21-\mathrm{v}\end{array}$ & $\begin{array}{l}\text { Comptes du palais, } \\
\text { du château et du bateau } \\
\text { de Poitiers }\end{array}$ & Étienne Gervais & 11-fév-87 & 29-fév-88 \\
\hline 6 & $\begin{array}{l}\text { BnF, ms fr. } 20686 \text {, } \\
\text { fol. } 22-25 \mathrm{v}\end{array}$ & $\begin{array}{l}\text { Comptes du château } \\
\text { de Lusignan }\end{array}$ & ? & 23-sep-86 & ? \\
\hline 7 & $\begin{array}{l}\text { BnF, ms fr. } 20 \text { 686, } \\
\text { fol. 42-49v }\end{array}$ & $\begin{array}{l}\text { Comptes du château } \\
\text { de Lusignan }\end{array}$ & Jean Aubereau & 02-déc-99 & 29-nov-00 \\
\hline 8 & $\begin{array}{l}\text { BnF, ms fr. } 20686 \text {, } \\
\text { fol. } 50-57 \mathrm{v}\end{array}$ & $\begin{array}{l}\text { Comptes du château } \\
\text { de Lusignan }\end{array}$ & Jean Aubereau & 04-déc-02 & 26-nov-03 \\
\hline 9 & $\begin{array}{l}\text { BnF, ms fr. } 11488, \\
\text { fol. 2-5v et Arch. nat., } \\
\text { KK 255, fol. 1-46v }\end{array}$ & $\begin{array}{l}\text { Comptes du palais } \\
\text { de Riom }\end{array}$ & Jean de Savignon & 01-aoû-82 & 01-aoû-84 \\
\hline 10 & $\begin{array}{l}\text { BnF, ms fr. } 11488, \\
\text { fol. } 6-13 \mathrm{v}\end{array}$ & $\begin{array}{l}\text { Comptes du palais } \\
\text { de Riom }\end{array}$ & Jean de Savignon & 01-aoû-86 & 01-aoû-87 \\
\hline 11 & $\begin{array}{l}\text { BnF, ms fr. } 26027 \\
\text { fol. } 2296-2297 \mathrm{v}\end{array}$ & $\begin{array}{l}\text { Comptes du palais } \\
\text { de Riom }\end{array}$ & ? & 1395 & 1396 \\
\hline 12 & $\begin{array}{l}\text { BnF, ms fr. } 26023, \\
\text { fol. } 1348-1353 \mathrm{v}\end{array}$ & $\begin{array}{l}\text { Comptes du château } \\
\text { d'Usson }\end{array}$ & Jean Bouton & 07-déc- 88 & 24-mai-89 \\
\hline 13 & $\begin{array}{l}\text { BnF, ms fr. } 20 \text { 686, } \\
\text { fol. 8-v }\end{array}$ & $\begin{array}{l}\text { Comptes du palais } \\
\text { de Bourges et du château } \\
\text { de Mehun-sur-Yèvre }\end{array}$ & $\begin{array}{l}\text { Huguenin } \\
\text { Meschin }\end{array}$ & 21-mai-80 & 20-mai-81 \\
\hline 14 & $\begin{array}{l}\text { Arch. Dép., Cher, } \\
8 \text { G 1166, fol. 1-4v }\end{array}$ & $\begin{array}{l}\text { Comptes du palais } \\
\text { de Bourges et du château } \\
\text { de Mehun-sur-Yèvre }\end{array}$ & $\begin{array}{l}\text { Huguenin } \\
\text { Meschin }\end{array}$ & 21-mai-81 & 21-mai-82 \\
\hline 15 & $\begin{array}{l}\text { BnF, ms fr. } 20686 \text {, } \\
\text { fol. } 58-59 v\end{array}$ & $\begin{array}{l}\text { Comptes du château } \\
\text { du Mehun-sur-Yèvre }\end{array}$ & $\begin{array}{l}\text { Jean } \\
\text { de Chalemaigne }\end{array}$ & 01-oct- 07 & 30-sep-08 \\
\hline
\end{tabular}

Tableau 5 : Les comptes des payeurs des œuvres de Jean de Berry (1380-1408), seul le compte $n^{\circ} 5$ est complet.

\begin{tabular}{|c|l|l|c|l|c|c|c|}
\hline \multicolumn{2}{|c|}{ Comptes } & \multicolumn{2}{c|}{$\begin{array}{c}\text { Datation } \\
\text { des exercices }\end{array}$} & \multicolumn{2}{c|}{ Estimation des lacunes } \\
\hline \multicolumn{1}{|c|}{ Désignation } & $\begin{array}{c}\text { Payeur des } \\
\text { ouvres } \\
\text { Début } \\
\text { (ns) }\end{array}$ & Fin (ns) & $\begin{array}{c}\text { Nombre } \\
\text { de } \\
\text { feuillets } \\
\text { du } \\
\text { compte } \\
\text { original }\end{array}$ & $\begin{array}{c}\text { Nombre } \\
\text { de } \\
\text { feuillets } \\
\text { conservés }\end{array}$ & Lacunes \\
\hline 1 & $\begin{array}{l}\text { Comptes du palais, } \\
\text { du château et du bateau } \\
\text { de Poitiers }\end{array}$ & $\begin{array}{l}\text { Étienne } \\
\text { Gervais }\end{array}$ & 09 -fév-83 & 07 -fév-84 & ? & 8 & $?$ \\
\hline 2 & $\begin{array}{l}\text { Comptes du palais, } \\
\text { du château et du bateau } \\
\text { de Poitiers }\end{array}$ & $\begin{array}{l}\text { Étienne } \\
\text { Gervais }\end{array}$ & 08 -fév-84 & 12 -fév-85 & 104 & 60 & 44 \\
\hline
\end{tabular}




\begin{tabular}{|c|c|c|c|c|c|c|c|}
\hline 3 & $\begin{array}{l}\text { Comptes du palais, } \\
\text { du château et du bateau } \\
\text { de Poitiers }\end{array}$ & $\begin{array}{l}\text { Étienne } \\
\text { Gervais }\end{array}$ & 13-fév-85 & 11-fév-86 & 62 & 39 & 23 \\
\hline 4 & $\begin{array}{l}\text { Comptes du palais, } \\
\text { du château et du bateau } \\
\text { de Poitiers }\end{array}$ & $\begin{array}{l}\text { Étienne } \\
\text { Gervais }\end{array}$ & 12-fév-86 & 10 -fév-87 & 85 & 85 & 0 \\
\hline 5 & $\begin{array}{l}\text { Comptes du palais, } \\
\text { du château et du bateau } \\
\text { de Poitiers }\end{array}$ & $\begin{array}{l}\text { Étienne } \\
\text { Gervais }\end{array}$ & 11-fév-87 & 29-fév-88 & $?$ & 1 & $?$ \\
\hline 6 & $\begin{array}{l}\text { Comptes du château } \\
\text { de Lusignan }\end{array}$ & ? & 23 -sep-86 & $?$ & $?$ & 4 & $?$ \\
\hline 7 & $\begin{array}{l}\text { Comptes du château } \\
\text { de Lusignan }\end{array}$ & $\begin{array}{l}\text { Jean } \\
\text { Aubereau }\end{array}$ & 02-déc-99 & 29-nov-00 & ? & 8 & $?$ \\
\hline 8 & $\begin{array}{l}\text { Comptes du château } \\
\text { de Lusignan }\end{array}$ & \begin{tabular}{|l} 
Jean \\
Aubereau
\end{tabular} & 04-déc-02 & 26-nov- 03 & $?$ & 8 & ? \\
\hline 9 & $\begin{array}{l}\text { Comptes du palais } \\
\text { de Riom }\end{array}$ & $\begin{array}{l}\text { Jean } \\
\text { de Savignon }\end{array}$ & $\mid 01$-aoû-82 & 01-aoû-84 & $64(?)$ & 51 & $13(?)$ \\
\hline 10 & $\begin{array}{l}\text { Comptes du palais } \\
\text { de Riom }\end{array}$ & $\begin{array}{l}\text { Jean } \\
\text { de Savignon }\end{array}$ & 01-aoû-86 & 01 -aoû- 87 & $?$ & 8 & $?$ \\
\hline 11 & $\begin{array}{l}\text { Comptes du palais } \\
\text { de Riom }\end{array}$ & $?$ & 1395 & 1396 & $?$ & 2 & $?$ \\
\hline 12 & $\begin{array}{l}\text { Comptes du château } \\
\text { d'Usson }\end{array}$ & Jean Bouton & 07-déc-88 & 24-mai-89 & $?$ & 6 & $?$ \\
\hline 13 & \begin{tabular}{|l|} 
Comptes du palais \\
de Bourges et du château \\
de Mehun-sur-Yèvre
\end{tabular} & $\begin{array}{l}\text { Huguenin } \\
\text { Meschin }\end{array}$ & 21-mai-80 & 20-mai-81 & $?$ & 1 & ? \\
\hline 14 & $\begin{array}{l}\text { Comptes du palais } \\
\text { de Bourges et du château } \\
\text { de Mehun-sur-Yèvre }\end{array}$ & $\begin{array}{l}\text { Huguenin } \\
\text { Meschin }\end{array}$ & 21-mai-81 & 21-mai-82 & ? & 4 & $?$ \\
\hline 15 & $\begin{array}{l}\text { Comptes du château } \\
\text { du Mehun-sur-Yèvre }\end{array}$ & $\begin{array}{l}\text { Jean de } \\
\text { Chalemaigne }\end{array}$ & 01-oct-07 & 30 -sep-08 & $?$ & 2 & $?$ \\
\hline & \multicolumn{4}{|l|}{ Total } & $?$ & 287 & ? \\
\hline
\end{tabular}

Tableau 6 : État des comptes des payeurs des œuvres (nombre de feuillets).

\section{La disparition de comptes au sein d'une série}

Les périodes d'activité des chantiers de Jean de Berry établies à partir des sources judiciaires, notariales ou des chroniques ne sont que partiellement couvertes par les sources comptables conservées, que ce soit les comptes ou les pièces justificatives. Certaines séries de comptes sont très incomplètes comme celle de Jean Aubereau, payeur des œuvres du château de Lusignan. Nous possédons pour ce château deux comptes, le premier allant du 2 décembre 1399 au 29 novembre $1400^{85}$, le suivant du 4 décembre 1402 au 26 novembre $1403^{86}$ (tableau 7). L'étude de ces deux épaves

85. BnF, ms fr. 20686 , fol. $42-49 \mathrm{v}$.

86. BnF, ms fr. 20686 , fol. $50-57 \mathrm{v}$. 


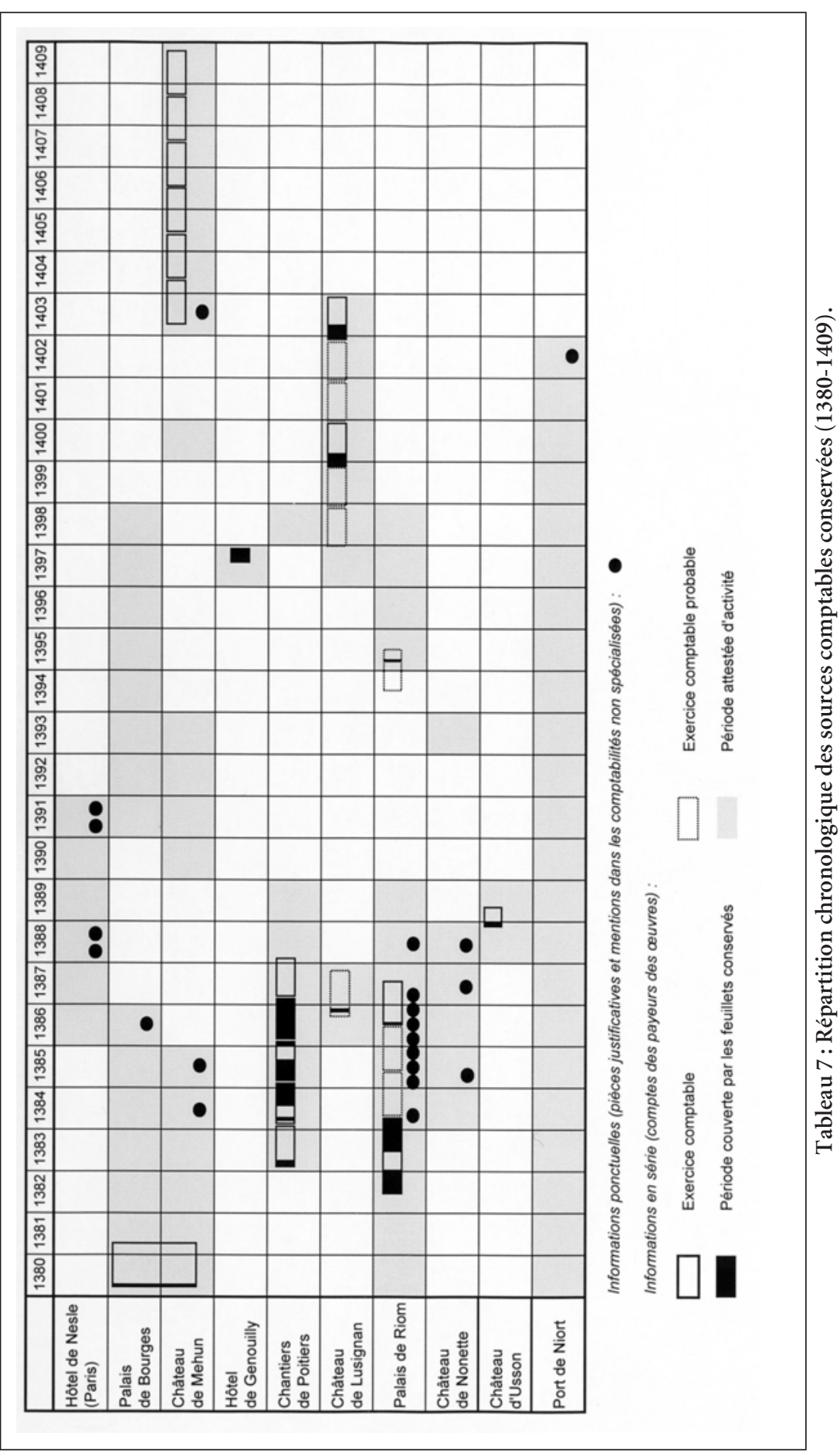

Tabularia «Études», nº 6, 2006, p. 33-73, 12 juillet 2006 
montre qu'elles présentent les mêmes caractéristiques externes et internes et qu'à l'évidence, deux comptes perdus les séparaient autrefois. En outre, une annotation latine portée au début du premier compte nous indique que le payeur des œuvres louait les services d'un procureur dès l'année 1397. Ces quelques informations permettent d'établir une chronologie a minima du chantier. Il existe une autre épave d'un compte anonyme rendu à la fin de l'année $1386^{87}$, mais elle ne montre aucun point commun avec les comptes de Jean Aubereau. Cette rupture peut signifier une probable phase d'inactivité entre deux campagnes de travaux distinctes.

Les épaves de comptes de Mehun-sur-Yèvre sont séparées, quant à elles, de presque trente ans (tableau 7) alors qu'un assez grand nombre de témoignages attestent que le chantier de cette résidence, qui revêtait aux yeux du prince une importance capitale, ne s'est pratiquement jamais interrompu. Trois séries de comptes ont complètement disparu : les comptes de l'hôtel de Nesle, du château de Nonette et du port de Niort. L'hôtel de Nesle est acquis en 1380. Cinq documents montrent que Jean de Berry fait entreprendre de grands travaux ${ }^{88}$. Par ailleurs, des archives judiciaires du XVII e siècle nous apprennent que la Chambre des comptes possédait une autre série de comptes comprise entre 1410 et 1418 pour des travaux concernant en partie cet hôtel ${ }^{89}$. Le chantier de Nonette fait partie des premiers grands travaux lancés par le prince: Guy de Dampmartin y travaille dès $1374^{90}$. On sait également, par une belle série de quittances, que ce chantier est actif de 1383 à $1389^{91}$. Le chantier du port de Niort est décidé dès 1377 mais il ne reste que deux documents prouvant l'existence d'un payeur des œuvres en $1403^{92}$.

\section{La disparition des feuillets dans les comptes conservés}

L'étude codicologique est particulièrement intéressante dans le cas de comptes incomplets ${ }^{93}$. Elle consiste à relever toutes les informations susceptibles de nous aider à reconstituer l'état ancien des comptes. Cette analyse comprend l'étude de la reliure, des feuillets de parchemin, de la justification du texte ainsi que des annotations laissées par les auditeurs.

Pour les comptes ayant perdu leurs premiers feuillets (tableau 9), il faut être en mesure de déterminer indirectement l'identité du payeur des œuvres ou de celui qui

87. BnF, ms fr. 20686 , fol. $22-25 \mathrm{v}$.

88. BnF, nouv. acq. fr., 7378, BnF, coll. Clairambault, vol. 4, pièces n ${ }^{\circ} 85$ et 86 ; BnF PO 55, pièces $\mathrm{n}^{\circ} 11$ et 12 .

89. Bibliothèque Historique de la Ville de Paris, 92 282, Sommaire des titres et des raisons qui prouvent que les murs et les fossez sur lesquels est baty le collège Mazarin n'estoient point des murs et des fossez de la ville de Paris, mais les fossez de l'hôtel de Nesle, lesquels appartenoient en priorité aux seigneurs dudit hôtel de Nesle, in-folio, 1688, 7 p. On trouve mentionné à la page 4 «Extraits des comptes rendus à la Chambre des comptes pour les années 1410-1411 jusqu'en 1418 de la dépense pour réparation aux tours, pont-levis, murs et l'hôtel de Nesle. Ils n'apparaissent pas dans les comptes de la ville [...]».

90. TeIlHARD DE CHARdin, «Registre... », p. 242-243.

91. BnF, PO 481, pièce $\mathrm{n}^{\circ} 12$; BnF, PO 702, pièce $\mathrm{n}^{\circ} 11 ; \mathrm{BnF}, \mathrm{PO} 2654$, pièces $\mathrm{n}^{\circ} 2-8$.

92. BnF, PO 678, pièces $n^{\circ} 2$ et 3 .

93. Concernant la codicologie, voir LEMAIRE, Jacques, Introduction à la codicologie, Louvain-La-Neuve, Institut d'études médiévales de l’Université catholique de Louvain, 1989. 
a tenu la comptabilité, la localisation du chantier et les termes de l'exercice. Cette tâche est compliquée pour les comptes réduits à quelques feuillets : il faut alors recourir à l'examen codicologique pour tenter un rapprochement avec une comptabilité déjà identifiée ou attendre de la collation du texte quelques indices. Malgré la mise en œuvre de telles observations, deux épaves restent anonymes ${ }^{94}$. La partie la plus importante d'un compte est celle réservée aux rubriques hebdomadaires (travaux en régie) retranscrites d'après les rôles tenus par le maître d'œuvre. La disparition de certaines de ces rubriques hebdomadaires entraîne d'importantes ruptures dans le suivi historique du chantier: l'ensemble des quinze comptes comptaient à l'origine 678 rubriques hebdomadaires : il n'en reste que 257 soit à peine $40 \%$ (tableau 8). Enfin, les derniers feuillets des comptes sont ceux qui furent le plus souvent perdus (tableau 9). Leur disparition implique celle de la documentation relative aux marchés baillés à tâche, des gages, des dons, des frais pour écriture, la balance du compte ainsi que la date d'audition du compte. Parmi les quinze registres, six ont conservé la totalité ou une partie de leurs chapitres pour ouvrages baillés à tâche, quatre leurs gages, cinq leurs balances générales. La perte des dépenses pour les travaux baillés à tâche est particulièrement gênante pour l'interprétation du compte. En effet, les comptes du château et du palais de Poitiers montrent que les marchés sont très importants: ils concernent souvent de grands ouvrages de terrassement, de maçonnerie ou de charpenterie et peuvent atteindre plus de la moitié de la dépense globale d'un exercice.

\begin{tabular}{|l|l|l|c|c|c|c|c|}
\hline $\mathrm{N}^{\circ}$ & \multicolumn{2}{|c|}{ Comptes } & \multicolumn{2}{c|}{$\begin{array}{c}\text { Datation } \\
\text { des exercices }\end{array}$} & \multicolumn{2}{c|}{$\begin{array}{l}\text { Estimation } \\
\text { des lacunes } \\
\text { (nb de rubriques } \\
\text { hebdo. }\end{array}$} \\
\hline 1 & $\begin{array}{l}\text { Comptes du palais, } \\
\text { du château et du bateau } \\
\text { de Poitiers }\end{array}$ & Étienne Gervais & 09 -fév-83 & 07 -fév-84 & 52 & 13 & 39 \\
\hline 2 & $\begin{array}{l}\text { Comptes du palais, } \\
\text { du château et du bateau } \\
\text { de Poitiers }\end{array}$ & Étienne Gervais & 08 -fév-84 & 12 -fév-85 & 52 & 23 & 29 \\
\hline 3 & $\begin{array}{l}\text { Comptes du palais, } \\
\text { du château et du bateau } \\
\text { de Poitiers }\end{array}$ & Étienne Gervais & 13 -fév-85 & 11 -fév-86 & 52 & 30 & 22 \\
\hline 4 & $\begin{array}{l}\text { Comptes du palais, } \\
\text { du château et du bateau } \\
\text { de Poitiers }\end{array}$ & Étienne Gervais & 12 -fév-86 & 10 -fév-87 & 52 & 52 & 0 \\
\hline 5 & $\begin{array}{l}\text { Comptes du palais, } \\
\text { du château et du bateau } \\
\text { de Poitiers }\end{array}$ & Étienne Gervais & 11 -fév-87 & 29 -fév-88 & 54 & 0 & 54 \\
\hline
\end{tabular}

94. BnF, ms fr. 20686, fol. 22-25v (compte du château de Lusignan commençant le 23 septembre 1386) ; BnF, ms fr. 26 027, fol. $2296-2297 v$ (compte de palais et de la Sainte-Chapelle de Riom daté de 1395 et 1396). 


\begin{tabular}{|c|l|l|c|c|c|c|c|}
\hline 6 & $\begin{array}{l}\text { Comptes du château } \\
\text { de Lusignan }\end{array}$ & $?$ & 23 -sep-86 & $?$ & $?$ & 4 & $?$ \\
\hline 7 & $\begin{array}{l}\text { Comptes du château } \\
\text { de Lusignan }\end{array}$ & Jean Aubereau & 02-déc-99 & 29 -nov-00 & 52 & 20 & 32 \\
\hline 8 & $\begin{array}{l}\text { Comptes du château } \\
\text { de Lusignan }\end{array}$ & Jean Aubereau & 04 -déc-02 & 26 -nov-03 & 52 & 22 & 30 \\
\hline 9 & $\begin{array}{l}\text { Comptes du palais } \\
\text { de Riom }\end{array}$ & $\begin{array}{l}\text { Jean } \\
\text { de Savignon }\end{array}$ & 01 -aoû-82 & 01 -aoû-84 & $81^{\text {a }}$ & 75 & 5 \\
\hline 10 & $\begin{array}{l}\text { Comptes du palais } \\
\text { de Riom }\end{array}$ & $\begin{array}{l}\text { Jean } \\
\text { de Savignon }\end{array}$ & 01 -aoû-86 & 01 -aoû-87 & 52 & 4 & 48 \\
\hline 11 & $\begin{array}{l}\text { Comptes du palais } \\
\text { de Riom }\end{array}$ & ? & 1395 & 1396 & $?$ & 3 & $?$ \\
\hline 12 & $\begin{array}{l}\text { Comptes du château } \\
\text { d’Usson }\end{array}$ & Jean Bouton & 07 -déc-88 & 24 -mai-89 & 23 & 2 & 21 \\
\hline 13 & $\begin{array}{l}\text { Comptes du palais } \\
\text { de Bourges et } \\
\text { du château de Mehun- } \\
\text { sur-Yèvre }\end{array}$ & $\begin{array}{l}\text { Huguenin } \\
\text { Meschin }\end{array}$ & 21 -mai-80 & 20 -mai-81 & 52 & 1 & 51 \\
\hline 14 & $\begin{array}{l}\text { Comptes du palais } \\
\text { de Bourges et } \\
\text { du château } \\
\text { de Mehun-sur-Yèvre }\end{array}$ & $\begin{array}{l}\text { Huguenin } \\
\text { Meschin }\end{array}$ & 21 -mai-81 & 21 -mai-82 & 52 & 8 & 44 \\
\hline 15 & $\begin{array}{l}\text { Comptes du château } \\
\text { du Mehun-sur-Yèvre }\end{array}$ & $\begin{array}{l}\text { Jean de } \\
\text { Chalemaigne }\end{array}$ & 01 -oct-07 & 30 -sep-08 & 52 & 0 & 52 \\
\hline & 678 & 257 & 428 & & & \\
\hline
\end{tabular}

Tableau 8: État des rubriques hebdomadaires dans les comptes des payeurs des œuvres ${ }^{\mathrm{b}}$.

a. Ce compte ne contenait que 81 rubriques hebdomadaires car les travaux en régie commencent seulement le 5 janvier 1383 .

b. La durée exacte des exercices des comptes n ${ }^{\circ} 6$ et 11 n'étant pas connue, ces derniers n'entrent pas dans le calcul du tableau $n^{\circ} 8$.

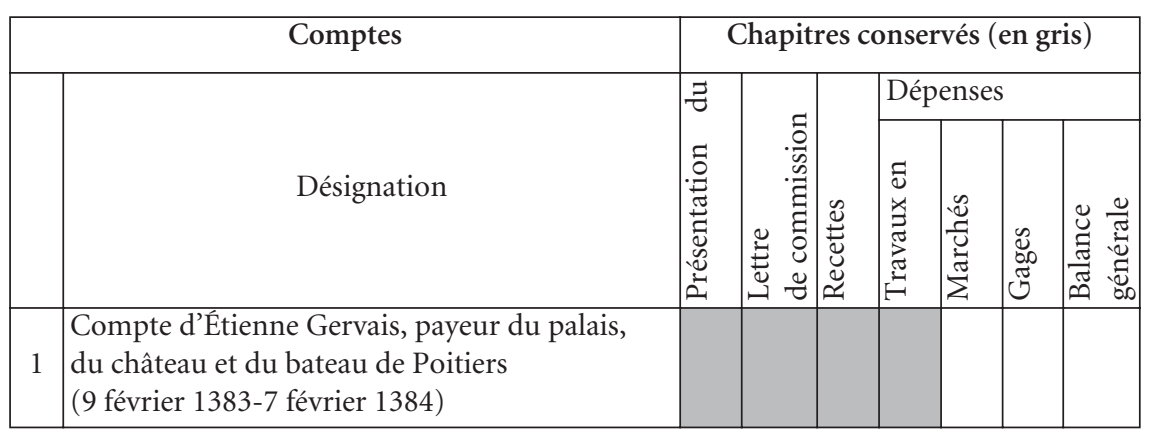




\begin{tabular}{|c|c|c|c|c|c|c|c|c|}
\hline 2 & $\begin{array}{l}\text { Compte d'Étienne Gervais, payeur du palais, } \\
\text { du château et du bateau de Poitiers } \\
\text { ( } 8 \text { février 1384-12 février 1385) }\end{array}$ & & & & & & & \\
\hline 3 & $\begin{array}{l}\text { Compte d'Étienne Gervais, payeur du palais, } \\
\text { du château et du bateau de Poitiers } \\
\text { (13 février-1385-11 février 1386) }\end{array}$ & & & & & & & \\
\hline 4 & $\begin{array}{l}\text { Compte d'Étienne Gervais, payeur du palais, } \\
\text { du château et du bateau de Poitiers } \\
(12 \text { février 1386-10 février 1387) }\end{array}$ & & & & & & & \\
\hline 5 & $\begin{array}{l}\text { Compte d'Étienne Gervais, payeur du palais, } \\
\text { du château et du bateau de Poitiers } \\
\text { (11 février 1387-29 février 1388) }\end{array}$ & & & & & & & \\
\hline 6 & $\begin{array}{l}\text { Compte du payeur du château de Lusignan } \\
\text { (23 septembre 1386-?) }\end{array}$ & & & & & & & \\
\hline 7 & $\begin{array}{l}\text { Compte de Jean Aubereau, payeur du château } \\
\text { de Lusignan } \\
\text { ( } 2 \text { décembre } 1399-29 \text { novembre } 1400 \text { ) }\end{array}$ & & & & & & & \\
\hline 8 & $\begin{array}{l}\text { Compte de Jean Aubereau, payeur du château } \\
\text { de Lusignan } \\
\text { (4 décembre 1402-26 novembre 1403) }\end{array}$ & & & & & & & \\
\hline 9 & $\begin{array}{l}\text { Compte de Jean de Savignon, payeur du palais } \\
\text { de Riom } \\
\left(1^{\text {er }} \text { août } 1382-1^{\text {er }} \text { août } 1384\right)\end{array}$ & & & & & & & * \\
\hline 10 & $\begin{array}{l}\text { Compte de Jean de Savignon, payeur du palais } \\
\text { de Riom } \\
\left(1^{\text {er }} \text { août } 1386-1^{\text {er }} \text { août } 1387\right)\end{array}$ & & & & & & & \\
\hline 11 & $\begin{array}{l}\text { Compte du payeur du palais de Riom } \\
(1395-1396)\end{array}$ & & & & & & & \\
\hline 12 & $\begin{array}{l}\text { Compte de Jean Bouton, payeur du château } \\
\text { d’Usson } \\
\text { (7 décembre 1388-24 mai 1389) }\end{array}$ & & & & & & & \\
\hline 13 & $\begin{array}{l}\text { Compte de Huguenin Meschin, payeur du palais } \\
\text { de Bourges et du château de Mehun-sur-Yèvre } \\
\text { (21 mai 1380-20 mai 1381) }\end{array}$ & & & & & & & \\
\hline 14 & $\begin{array}{l}\text { Compte de Huguenin Meschin, payeur du palais } \\
\text { de Bourges et du château de Mehun-sur-Yèvre } \\
\text { (21 mai 1381-21 mai 1382) }\end{array}$ & & & & & & & \\
\hline 15 & $\begin{array}{l}\text { Compte de Jean de Chalemaigne, payeur } \\
\text { du château de Mehun-sur-Yèvre } \\
\left(1^{\text {er }} \text { octobre } 1407-30 \text { septembre } 1408\right)\end{array}$ & & & & & & & \\
\hline & Totaux & 11 & 2 & 10 & 13 & 6 & 4 & 5 \\
\hline
\end{tabular}

Tableau 9: État des chapitres dans les comptes des payeurs des œuvres. * balance sur l'exercice 1382-1383 uniquement.

La reconstitution du compte du payeur des œuvres du palais de Riom, Jean de Savignon ( $\mathrm{n}^{\circ} 9$ dans les tableaux 5, 6, 8 et 9), est un bon exemple de la contribution 
de la codicologie ${ }^{95}$. Aujourd'hui, ce compte est divisé en deux parties: un cahier inséré dans le manuscrit français 11488 de la BnF, et sept cahiers conservés aux Archives nationales sous la cote KK 255 (tableau 10). Les cahiers des Archives nationales sont toujours protégés par la couverture originale du registre, faite de cuir foncé. Une étiquette de parchemin indique: "Ce sont lez comptes Jehan de Savignon payeur des œuvres du palais de Riom qui contiennent deux ans commençant le premier jour d'ahoust l'an mil IIIc IIIIxx et deux et finissent semblable jour l'an mil IIIc IIIIxx et quatre». Les quatre feuillets issus du manuscrit français 11488 de la BnF proviennent du premier cahier du compte : les caractères codicologiques et la collation du texte le prouvent. Ce cahier a vraisemblablement perdu ses deux bifeuillets internes comme le montre une interruption dans le texte entre les actuels feuillets $3 \mathrm{v}$ et 4 (fig. 2). Les premiers feuillets (fol. $2 \mathrm{v}-3 \mathrm{v}$ ) enregistrent des achats de matériaux et quelques menus travaux alors qu'au feuillet 4 débutent véritablement les travaux en régie (à partir du 5 janvier 1383). Cette épave de cahier s'interrompt dans la semaine du 2 février 1383 (fol. 5v). Les 46 feuillets du KK 255 forment le reste du compte. On peut vérifier, grâce aux chiffres romains minuscules portés en haut des premiers feuillets de chacun des cahiers que ceux-ci sont encore reliés dans le bon ordre ${ }^{96}$. Cependant, certains de ces cahiers comportent des lacunes peu visibles sans un examen détaillé.

Le premier cahier du KK 255 (fol. 1-6v) était en fait le deuxième du compte (tableau 10). Il est composé seulement de trois bifeuillets, ce qui laisse supposer la soustraction d'un bifeuillet. L'observation des feuillets et la collation du texte de ce cahier montrent que c'est le bifeuillet extérieur qui manque (fig. 2). Effectivement, on note l'absence de chiffre romain sur le premier feuillet et la parfaite cohérence des données (texte et sommes) portées au centre du cahier. Enfin, la règle des visà-vis suppose que les cahiers commencent par un feuillet présentant sa face la plus lisse et la plus claire (face chair) alors que l'aspect de ce premier feuillet est sombre et rugueux (face poil) ${ }^{97}$. S'agissant d'un cahier privé de son bifeuillet extérieur, les données manquantes étaient portées sur le premier et le dernier feuillet de ce cahier. Le troisième cahier du compte (fol. 7-14v) est également un cahier modifié (fig. 2) ${ }^{98}$. Il se présente sous la forme de trois bifeuillets reliés auxquels sont ajoutés deux feuillets volants (fol. 13 et 14) solidaires des précédents par l'intermédiaire de leurs talons qui apparaissent au lecteur au recto du premier feuillet du cahier. Le premier de ces talons (pendant du feuillet 14) est grossièrement découpé et déborde d'environ $1,5 \mathrm{~cm}$ de la reliure. Le deuxième (pendant du feuillet 13) est découpé net à $1 \mathrm{~cm}$ de la reliure rabattu et collé au premier cahier (fol. 7). Ces deux feuillets isolés ne sont visiblement pas de même nature: le feuillet 13 respecte la règle des vis-à-vis

95. BnF ms fr. 11488 , fol. 2-5v, KK 255, fol. 1-46v (compte de Jean de Savignon, $1^{\text {er }}$ août $1382-1^{\text {er }}$ août 1384).

96. Il ne s'agit pas d'une foliotation mais d'une numérotation des cahiers afin de permettre leur reliure dans le bon ordre.

97. Règle de Gregory déterminant l'alternance des côtés poil et chair des feuillets de parchemin. Elle est appliquée dans l'ensemble du compte.

98. Deuxième cahier du KK 255. 
et constitue ce qui reste du bifeuillet extérieur de ce cahier. Ainsi, son pendant disparu formait le premier feuillet de ce cahier ${ }^{99}$. En revanche, le feuillet 14 ne respecte pas la règle des vis-à-vis et le piquage de réglure, décalé par rapport au verso du feuillet 13, montre qu'il s'agit visiblement d'un ajout ultérieur. Il faut conclure que ce troisième cahier a perdu son premier feuillet. Cette soustraction concerne le feuillet qui marquait le commencement de l'exercice 1383-1384 car celui-ci comportait la présentation du compte, les modalités de versement des recettes affectées au chantier ainsi qu'une partie des dépenses de la semaine du 3 août 1383. Cette perte n'est pas compensée par l'ajout du feuillet 14 car les informations portées sur ce dernier suivent parfaitement celles du feuillet 13 et précédent exactement celles du feuillet $15^{100}$. À l'origine, ce cahier devait donc présenter une forme hybride: un cahier augmenté d'un feuillet. Les trois cahiers suivants (14-38v), respectivement quatrième, cinquième et sixième cahiers du compte originel ${ }^{101}$, sont tous des cahiers complets (tableau 10). Les chiffres romains conservés permettent de les placer sans difficulté dans l'ordre du compte. Il faut signaler que le quatrième cahier (fol. 14-22v) contient le récapitulatif des six premiers mois de l'exercice sous le terme prima grossa ${ }^{102}$. Le septième cahier (fol. 39-45v) est un cahier incomplet. Il est marqué d'un «VII» ce qui signifie que ce cahier débute bien par son premier feuillet. La règle des vis-à-vis est conservée dans ce cahier sauf entre les feuillets 41 et $42^{103}$. En fait le feuillet 41 constitue le premier pendant de l'avant dernier bifeuillet alors que le 42 appartenait au bifeuillet interne. Le pendant de ce feuillet a été coupé et on a laissé $1 \mathrm{~cm}$ de talon pris dans la couture. Cette lacune est confirmée par la collation du texte: on passe sans transition ni récapitulatif des sommes de la semaine du 26 juin 1384 (fol. 41) à celle du 4 juillet (fol. 42) ${ }^{104}$. En lieu et place du huitième cahier, on trouve un simple feuillet isolé (fol. 46) relié avec les autres cahiers par un talon rabattu. Il s'agit du premier feuillet de ce huitième cahier disparu car il est numéroté «VIII ». Le recto conclut la secunda grossa, c'est-à-dire les travaux en régie pour l'exercice 1383-1384. Au verso commencent les dépenses pour marchés. L'unique marché retranscrit n'est pas complet et se poursuivait sur les feuillets suivants.

Le compte de Jean de Savignon pour l'exercice 1382-1384 n'est donc pas aussi complet qu'il y parait au premier abord. Il faut ajouter aux quatre feuillets perdus $\mathrm{du}$ premier cahier (Bnf, ms fr. 11 488, fol. 2-5v), les lacunes apparaissant après examen dans le KK 255. Nous pouvons faire état de la disparition de quatre feuillets dans le corps même du compte auxquels s'ajoutent les sept appartenant vraisemblablement

99. Pour cette raison le fol. 7 ne comporte pas de signature et présente une face poil au lieu d'une face chair.

100. La somme récapitulative de la semaine du 2 novembre 1383, portée en haut du fol. 14, donne la somme exacte des dépenses inscrites au feuillet 13. Il n'y a donc pas de perte d'informations. La semaine du 9 novembre s'achève au verso du feuillet 13 et celle du 17 novembre commence sur le feuillet 15 .

101. Ils sont les troisième, quatrième et cinquième du KK 255.

102. Arch. nat., KK 255, fol. 20v.

103. Le verso du feuillet 41 présente son côté poil alors qu'au contraire le recto du feuillet 42 est du côté chair.

104. L'interruption intervient au milieu d'un long chapitre consacré aux journées de charretiers. 


\begin{tabular}{|l|c|c|c|c|c|c|c|c|}
\hline Dépôt & $\begin{array}{c}\text { BnF ms fr. } \\
11488\end{array}$ & \multicolumn{8}{|c|}{ Arch. nat., KK 255 } \\
\hline Folio & $2-5 \mathrm{v}$ & $1-6 \mathrm{v}$ & $7-14 \mathrm{v}$ & $15-22 \mathrm{v}$ & $13-30 \mathrm{v}$ & $31-38 \mathrm{v}$ & $39-45 \mathrm{v}$ & $46-\mathrm{v}$ \\
\hline Cahier $\mathrm{n}^{\circ}$ & 1 & 2 & 3 & 4 & 5 & 6 & 7 & 8 \\
\hline État & & & & & & \\
\hline
\end{tabular}

Tableau 10 : Reconstitution du premier compte de Jean de Savignon ( $1^{\text {er }}$ août $1382-1^{\text {er }}$ août 1384$)$.

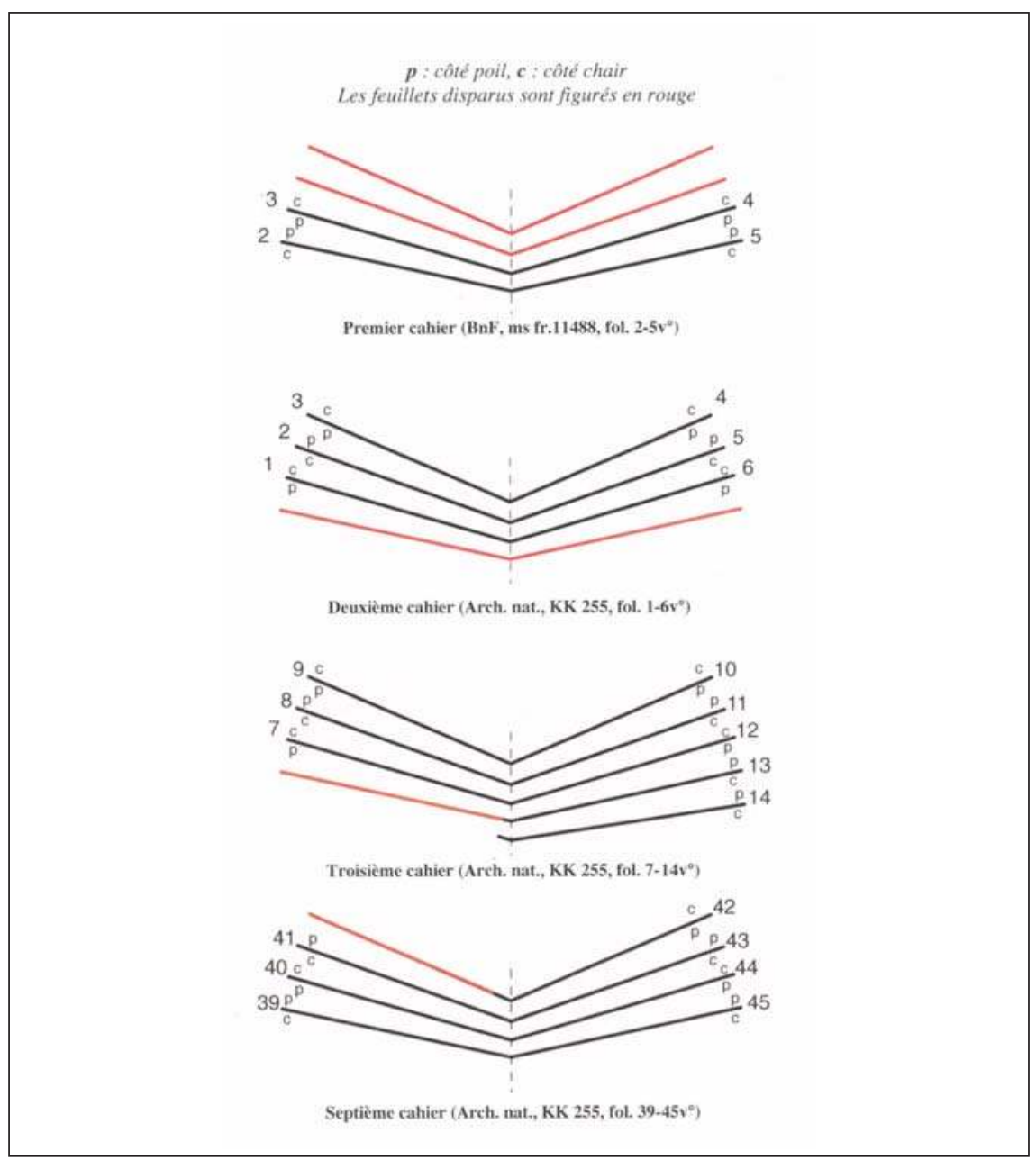

Figure 2: Schéma des quatre premiers cahiers incomplets du compte de Jean de Savignon payeur des œuvres du palais de Riom, du $1^{\text {er }}$ août 1382 au $1^{\text {er }}$ août 1384. $p$ : côté poil, $c$ : côté chair, les feuillets disparus sont figurés en rouge. 
au huitième cahier. Les feuillets soustraits du compte sont donc au nombre de quinze. Si nous les ajoutons aux cinquante feuillets conservés nous obtenons bien le nombre de feuillets contenus dans les huit quaternions ${ }^{105}$. Les rubriques hebdomadaires ne sont pas au nombre de 104 (deux années) car elles commencent seulement le 5 janvier 1383. Sur les 29 semaines comprises entre cette date et la fin de l'exercice ( $1^{\text {er }}$ août 1383), il subsiste 23 rubriques hebdomadaires. Malgré les quelques feuillets retirés dans le KK 255, toutes les semaines de l'exercice 1383-1384 sont enregistrées, soit 52. Ainsi, sur les 81 semaines comprises entre le 5 janvier 1383 et le premier août 1384, 75 rubriques hebdomadaires sont encore conservées intégralement (tableau 8).

\section{Les procédés variables de transcription du payeur des œuvres}

Il faut rappeler que les comptes des payeurs des ouvres synthétisent le contenu des pièces produites sur les chantiers. Il est logique qu'une grande quantité d'informations contenues dans ces pièces n'ait pas été reportée dans les comptes car jugée superflue par le payeur. Certains payeurs vont d'ailleurs réduire jusqu'à l'extrême la teneur des pièces. Par exemple, dans la rubrique «euvres» d'un compte de la terre de Graçay, le comptable rédige un seul article pour d'importantes réparations à l'hôtel de Genouilly s'élevant à la somme de $176 \mathfrak{E}$ t. et 18 sous ${ }^{106}$. Cet article renvoie aux "parties écrites en un quaier [de] 6 feuillez de papier [où] sont contenues les journées et sepmaines des ouvriers ». Ce cahier, unique témoin du chantier rédigé par le maître d'œuvre, n'a malheureusement pas été conservé. De surcroît, les payeurs étant également rémunérés au feuillet, il est probable que la Chambre des comptes leur a demandé de réduire la teneur de leurs articles par souci d'économie. Au milieu du XV $\mathrm{XV}^{\mathrm{e}}$ siècle, un capitaine de Lusignan rend un compte pour travaux et explique en préambule qu'il a rédigé son compte selon des directives lui prescrivant de faire «ung estat abregié» pour «abregier l'expedicion» du compte et pour éviter que «les fraiz de la façon du dit compte qui seroit grans se (sic) toutes les dites singulieres parties estoient escriptes au long ${ }^{107}$. Il est fort probable que ce genre d'injonctions a été fait aux payeurs des œuvres de Jean de Berry.

Ces différences de procédures posent un vrai problème à l'historien car un payeur peut avoir utilisé plusieurs procédés de transcription à l'intérieur d'une même série de comptes. C'est le cas d'Étienne Gervais, clerc payeur des œuvres du château et du palais de Poitiers, qui passe entre 1383 et 1388 d'une forme de rédaction développée à une forme synthétique ${ }^{108}$.

La forme de rédaction développée facilitait le travail des examinateurs de la Chambre des comptes en leur permettant de vérifier l'identité des ouvriers ainsi que le montant de leur salaire, la nature et la durée de leur service (fig. 3). Ces chapitres

105. Un quaternion est un cahier de 4 bifeuillets. Le total de feuillets est de 65 c'est-à-dire 8 quaternions dont un est «augmenté » d'un feuillet (le troisième).

106. Bourges, Arch. dép. Cher, 8 G 2129, fol. 79v, Compte de la terre de Graçay (1410-1411).

107. BnF, ms fr. 6737 , fol. 35v (compte de messire Yves du Fou, chevalier et capitaine de Lusignan, ordonné à distribuer la somme de $2000 \mathfrak{E}$ tournois pour les réparations du dit château, 1er octobre 1463 - 31 septembre 1464).

108. BnF, ms fr. 20 686, fol. 13-20v; BnF, ms fr. 20 686, fol. 9-12v; Arch. nat., KK 256, fol. 1-56v; Arch. nat., KK 257a, fol. 1-39v; Arch. nat., KK 257b, fol. 1-85v; BnF, ms fr. 20 686, fol. 21 -v. 


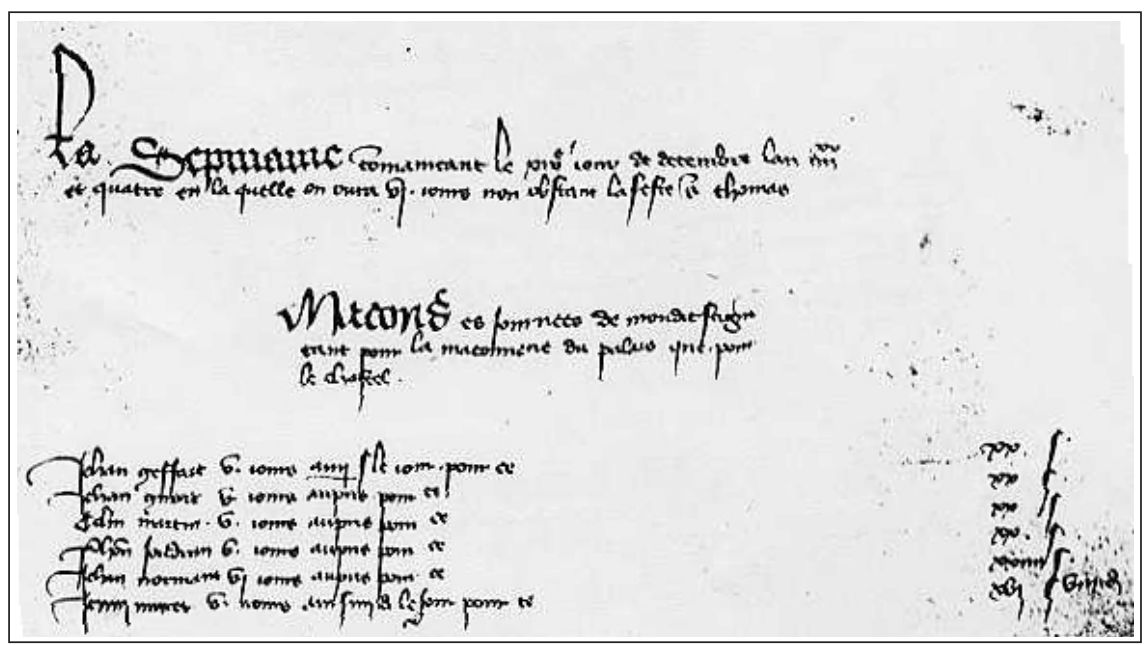

Figure 3: Exemple de transcription développée. Journées de maçons, compte d'Étienne Gervais, payeur des œuvres du palais et du château de Poitiers ( 9 février 1383 - 12 février 1385). Arch. nat., KK 256, fol. 37.

développés nous autorisent à entrevoir le détail de la composition des équipes et suivre le parcours des individus. Ce type de compte contient les données pour une analyse fine de la chronologie du chantier. Dans le tableau 11 ci-dessous (nombre de journées de maçons et tailleurs de pierres par semaine), on remarque par exemple l'accroissement de l'activité occasionné par la visite du prince en novembre 1384. Durant cette période, on compte jusqu'à 94 journées ainsi que deux nuitées nécessaires pour préparer sa résidence dans le château de Poitiers. L'activité, qui était retombée en décembre, reprend en janvier 1385 pour réaliser les gabarits de la tour Maubergeon (palais) dont le chantier commence à partir du 30 janvier. En outre, la mise en tableau nous permet de constater également la non permanence des équipes: on peut regrouper les hommes dans quatre équipes distinctes.

Le choix d'une rédaction développée pose de sérieux problèmes matériels au payeur car si l'activité prend de l'importance, le compte devient vite volumineux. Le compte allant du 8 février 1384 au 12 février 1385 était le plus long des quinze comptes avec plus d'une centaine de feuillets.

À partir de février 1385, Étienne Gervais prend le parti de synthétiser toutes les données des chantiers du palais et du château dans des chapitres très courts renvoyant les correcteurs de la Chambre des comptes aux pièces justificatives pour connaître l'identité des personnes, leur salaire et la nature de leur service (fig. 4). Le payeur des œuvres note simplement le montant global de la somme versée. Dans ces conditions, l'identification du personnel présent sur le chantier de Poitiers devient impossible. La rédaction synthétique pose surtout le problème de l'exploitation de l'ensemble de la série des cinq comptes qui ne sont pas conçus de la même manière. L'unique moyen de comparaison passe par le recours à un critère de mesure transversal: la dépense hebdomadaire (tableau 12). 


\begin{tabular}{|c|c|c|c|c|c|c|c|c|c|c|c|c|c|c|c|c|c|c|c|}
\hline $\begin{array}{l}\text { Nom } \\
(\mathrm{T}) \text { : tailleur } \\
\text { de pierre } \\
(\mathrm{M}): \text { maçon }\end{array}$ & 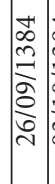 & 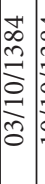 & & 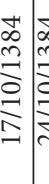 & & & & 至 & 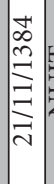 & & 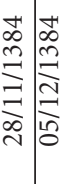 & 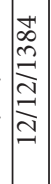 & 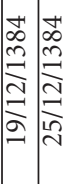 & 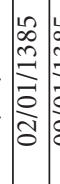 & & 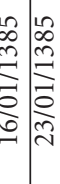 & 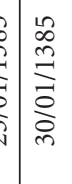 & 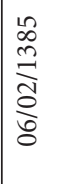 & 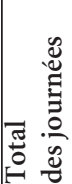 \\
\hline $\begin{array}{l}\text { Arnaut Perrot } \\
(\mathrm{M})\end{array}$ & & & & & & & & & & & & & & & & & & & 0 \\
\hline Avril Guillaume & & & & & & & \begin{tabular}{l|l}
6 & 6 \\
\end{tabular} & $\begin{array}{ll}5 & 1 \\
\end{array}$ & 6 & 2 & & 6 & & & & & 6 & 6 & 39 \\
\hline Aymaret Guyon & & & & & & & \begin{tabular}{l|l}
3 & 6
\end{tabular} & & 4 & & & & & & & & & & 13 \\
\hline $\begin{array}{l}\text { Bardon Pierre } \\
(\mathrm{M})\end{array}$ & & & & & & & & & & & & 6 & & & & & & & 6 \\
\hline Berton Jean le & & & & & & & & & & & & & & & & & 6 & 6 & 12 \\
\hline Blois Jean de $(\mathrm{T})$ & & & & & & & & & & & & & & & & & 6 & 6 & 12 \\
\hline $\begin{array}{l}\text { Bruges Henequin } \\
\text { de }\end{array}$ & & & & & & & & & & & & & & & & & 6 & 6 & 12 \\
\hline Caillou Jean & & & & & & & & & 6 & & & & & & & & 6 & 6 & 18 \\
\hline Charron Jean le & & & & & & & & & & & & & & & & & 6 & 6 & 12 \\
\hline Chilleon Jean & & & & & & & 6 & & & & & & & & & & & & 6 \\
\hline Cornille Micheau & & & & & & & & & & & & & & & & & 6 & 6 & 12 \\
\hline Davaille Jean (M) & & & & & & & & & & & & 6 & & & & & & & 6 \\
\hline Doe Richard (T) & & & & & & & & & & & & & & & & & & 6 & 6 \\
\hline Esmeri Jennin $(\mathrm{T})$ & & & & & & & & & & & & & & & & & & 6 & 6 \\
\hline $\begin{array}{l}\text { Flamant } \\
\text { Henequin le }\end{array}$ & & & & & & & & & & & & & & 6 & 6 & 6 & & & 18 \\
\hline \begin{tabular}{|l|} 
Fleau Micheau \\
\end{tabular} & & & & & & & & & & & & & & & & & 6 & 6 & 12 \\
\hline Garmeau Simon & & & & $6: 5$ & \begin{tabular}{l|l}
5 & 2 \\
\end{tabular} & \begin{tabular}{l|l}
4 & 6 \\
\end{tabular} & \begin{tabular}{l|l}
6 & 6 \\
\end{tabular} & \begin{tabular}{|l|l|}
5 & 3 \\
\end{tabular} & 6 & 2 & & & & & & & & & 38 \\
\hline Gessart Jean (M) & & & & & & & \begin{tabular}{l|l}
6 & 6 \\
\end{tabular} & \begin{tabular}{|l|l|}
5 & 4
\end{tabular} & 6 & 2 & & 6 & 5 & & 5 & & 6 & 6 & 52 \\
\hline Girart Jean (T) & & & & & & & & & \begin{tabular}{|l|l|}
6 \\
\end{tabular} & & & & 5 & & 5 & & 6 & 6 & 28 \\
\hline Girart Pierre $(\mathrm{T})$ & & & & & & & & & & & & & & & & & & 6 & 6 \\
\hline Gizay Perrot de & & & & & & & 6 & & 3 & & & & & & & & & & 9 \\
\hline Gordin Jean (T) & & & & & & & & & & & & & & & & & & 6 & 6 \\
\hline Guerin Perrot & & & & & & & 6 & & 6 & & & & & & & & & & 12 \\
\hline Huy Jean de & & & & & & & & & & & & & & 6 & 6 & 6 & & & 18 \\
\hline $\begin{array}{l}\text { Hetout Jacquet } \\
(\mathrm{T})\end{array}$ & & & & & & & & & & & & & & & & & 6 & 6 & 12 \\
\hline $\begin{array}{l}\text { Jarnac Etienne } \\
(\mathrm{M})\end{array}$ & 5 & 6 & \begin{tabular}{l|l}
6 & 6
\end{tabular} & 6 & 5 & 4 & & & & & & & & & & & & & 32 \\
\hline $\begin{array}{l}\text { Jourdain Phelip- } \\
\text { pon }\end{array}$ & & & & & & & 6 & & 6 & 2 & & & 5 & & & & & & 19 \\
\hline $\begin{array}{l}\text { Laverde Guillemin } \\
(\mathrm{T})\end{array}$ & & & & & & & & & & & & & & & & & 6 & & 6 \\
\hline Mache Jean & & & & & & & & & 6 & & & & & & & & & & 6 \\
\hline Martin Colin & & & & & & & & & 6 & & & 6 & 5 & & & & & & 17 \\
\hline Martin Perrot & & & & & & & & & \begin{tabular}{|l|l|}
6 & \\
\end{tabular} & & & & & & & & & & 6 \\
\hline May Perrin (T) & & & & & & & & & & & & & & & & & 6 & & 6 \\
\hline $\begin{array}{l}\text { Moreau Perrin } \\
(\mathrm{M})\end{array}$ & & & & & & & & & & & & 1 & & & & & & & 1 \\
\hline
\end{tabular}




\begin{tabular}{|c|c|c|c|c|c|c|c|c|c|c|c|c|c|c|c|c|c|c|c|c|c|c|}
\hline Muret Jean (M) & & & & & & & & 6 & & 6 & 4 & & & \begin{tabular}{l|l}
6 & 5
\end{tabular} & 5 & & & & & 6 & 6 & 39 \\
\hline \begin{tabular}{|l|} 
Negrier \\
Guillaume (M)
\end{tabular} & 5 & 6 & 6 & 6 & 5 & 4 & & & & & & & & & & & & & & & & 32 \\
\hline $\begin{array}{l}\text { Nevers Girart de } \\
(\mathrm{T})\end{array}$ & & & & & & & & & & & & & & & & & & & & 6 & 6 & 12 \\
\hline $\begin{array}{l}\text { Nevers Thévenin } \\
\text { de (T) }\end{array}$ & & & & & & & & & & & & & & & & & & & & 6 & 6 & 12 \\
\hline $\begin{array}{l}\text { Normant Jean } \\
\text { (M) }\end{array}$ & & & & & & & & & & & & & & 6 & 6 & & & & & & & 12 \\
\hline Pre Jacques du & & & & & & & & & & & & & & & & & & & & 6 & 6 & 12 \\
\hline Odin Étienne (M) & 5 & 6 & 6 & 6 & 6 & 4 & & & & 6 & & & & & & & & & & & & 39 \\
\hline Samier Jean (M) & & & & & & & & 6 & & 6 & 4 & & & 6 & & & & & & & & 22 \\
\hline Saussart Thevenin & & & & & & & 6 & 6 & & 6 & 2 & & & & & & & & & & & 20 \\
\hline $\begin{array}{l}\text { Jean de Vernoil } \\
\text { (M) }\end{array}$ & & & & & & & & & & 3 & & & & 6 & & & & & & & & 9 \\
\hline $\begin{array}{l}\text { Villier Guiot de } \\
(\mathrm{T})\end{array}$ & & & & & & & & & & & & & & & & & & & & 6 & 6 & 12 \\
\hline Total des journées & 15 & & 18 & & 42 & 16 & 27 & 66 & 8 & 94 & & 0 & 0 & & 31 & \begin{tabular}{l|l}
0 & 1
\end{tabular} & & 212 & 0 & 108 & 120 & 685 \\
\hline
\end{tabular}

Tableau 11: Nombre de journées de maçon et de tailleurs de pierre travaillant à Poitiers pendant les semaines allant de septembre 1384 à février 1385 d'après le compte Arch. nat., KK 256.

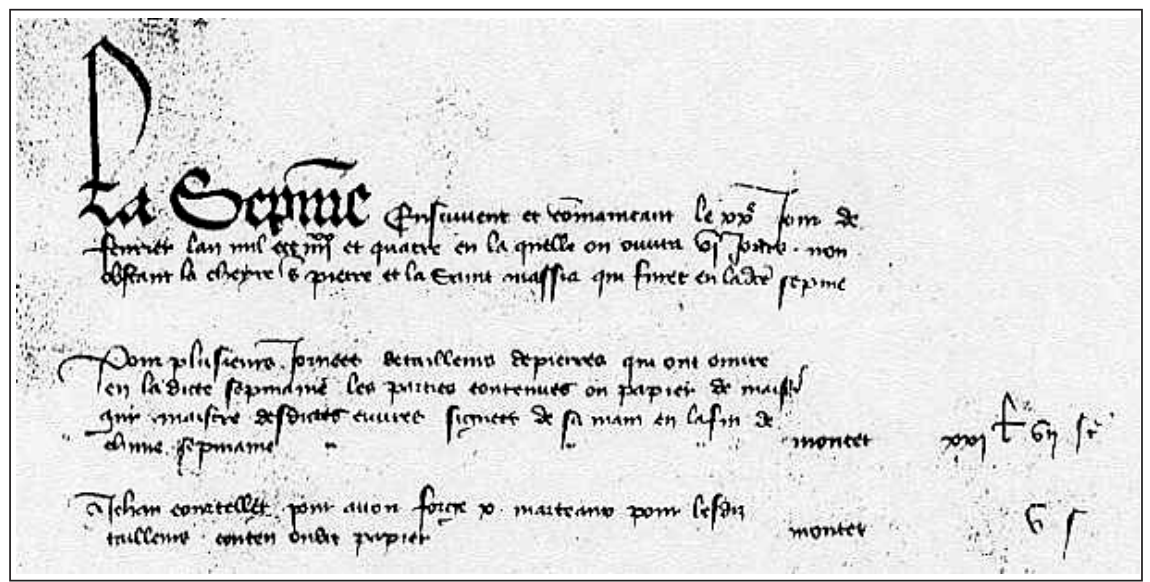

Figure 4: Exemple de transcription synthétique. Dépenses globales, compte d’Étienne Gervais, payeur des œuvres du palais et du château de Poitiers (12 février 1385 - 10 février 1386) Arch. nat., KK 257a, fol. 3v.

Ainsi la longueur matérielle d'un compte ne constitue pas un indicateur fiable de l'activité du chantier. Un compte court, du fait de l'utilisation d'une forme de rédaction synthétique (fig. 4), peut très bien révéler en fait une grande activité du chantier. Par exemple, le compte Arch. nat., KK 256 (Poitiers 1384 - 1385, fig. 3) comprend 104 feuillets et le compte Arch. nat., KK257a (Poitiers 1385 - 1386, fig. 4) n'en fait que 62 alors que les dépenses ont en fait augmenté entre ces deux exercices (tableau 12). 


\begin{tabular}{|c|c|c|c|c|c|}
\hline semaines & $\varepsilon$ & semaines & $\varepsilon$ & semaines & $\varepsilon$ \\
\hline $9 / 2 / 1383$ & & $20 / 2 / 1385$ & 84 & $23 / 4 / 1386$ & 39 \\
\hline $16 / 2 / 1383$ & & $27 / 2 / 1385$ & 81 & $30 / 4 / 1386$ & 132 \\
\hline $23 / 2 / 1383$ & & $6 / 3 / 1385$ & 116 & $7 / 5 / 1386$ & 151 \\
\hline $2 / 3 / 1383$ & & $13 / 3 / 1385$ & 76 & $14 / 5 / 1386$ & 140 \\
\hline $9 / 3 / 1383$ & & $20 / 3 / 1385$ & 74 & $21 / 5 / 1386$ & 342 \\
\hline $16 / 3 / 1383$ & & $27 / 3 / 1385$ & 64 & $28 / 5 / 1386$ & 111 \\
\hline $23 / 3 / 1383$ & & $3 / 4 / 1385$ & 52 & $4 / 6 / 1386$ & 142 \\
\hline $30 / 3 / 1383$ & & $10 / 4 / 1385$ & 136 & $11 / 6 / 1386$ & 55 \\
\hline $6 / 4 / 1383$ & 11 & $17 / 4 / 1385$ & 132 & $18 / 6 / 1386$ & 195 \\
\hline $13 / 4 / 1383$ & & $24 / 4 / 1385$ & 130 & $25 / 6 / 1386$ & 138 \\
\hline $20 / 4 / 1383$ & 8 & $1 / 5 / 1385$ & 115 & $2 / 7 / 1386$ & 167 \\
\hline $27 / 4 / 1383$ & 11 & $8 / 5 / 1385$ & 150 & $9 / 7 / 1386$ & 162 \\
\hline $4 / 5 / 1383$ & 7 & $15 / 5 / 1385$ & 156 & $16 / 7 / 1386$ & 128 \\
\hline $11 / 5 / 1383$ & 7 & $22 / 5 / 1385$ & 106 & $24 / 7 / 1386$ & 274 \\
\hline $18 / 5 / 1383$ & & $29 / 5 / 1385$ & 78 & $29 / 7 / 1386$ & 131 \\
\hline lacune & & $5 / 6 / 1385$ & 105 & $6 / 8 / 1386$ & 142 \\
\hline $8 / 2 / 1384$ & & $12 / 6 / 1385$ & 105 & $13 / 8 / 1386$ & 103 \\
\hline lacune & & $19 / 6 / 1385$ & 81 & $20 / 8 / 1386$ & 182 \\
\hline $29 / 2 / 1384$ & 27 & $26 / 6 / 1385$ & 27 & $27 / 8 / 1386$ & 134 \\
\hline $7 / 3 / 1384$ & & $3 / 7 / 1385$ & 115 & $3 / 9 / 1386$ & 141 \\
\hline lacune & & $10 / 7 / 1385$ & 90 & $10 / 9 / 1386$ & 169 \\
\hline $19 / 9 / 1384$ & 35 & $17 / 7 / 1385$ & 124 & $17 / 9 / 1386$ & 188 \\
\hline $26 / 9 / 1384$ & 58 & $24 / 7 / 1385$ & 99 & $23 / 9 / 1386$ & 180 \\
\hline $3 / 10 / 1384$ & 98 & $31 / 7 / 1385$ & 104 & $1 / 10 / 1386$ & 200 \\
\hline $10 / 10 / 1384$ & 56 & $7 / 8 / 1385$ & 92 & $8 / 10 / 1386$ & 174 \\
\hline $17 / 10 / 1384$ & 71 & $14 / 8 / 1385$ & 65 & $15 / 10 / 1386$ & 212 \\
\hline $24 / 10 / 1384$ & 51 & $21 / 8 / 1385$ & 71 & $22 / 10 / 1386$ & 136 \\
\hline $31 / 10 / 1384$ & 69 & $28 / 8 / 1385$ & 113 & $29 / 10 / 1386$ & 82 \\
\hline $7 / 111 / 1384$ & 118 & $4 / 9 / 1385$ & & \begin{tabular}{|l|}
$5 / 10 / 1386$ \\
\end{tabular} & 163 \\
\hline $14 / 11 / 11384$ & 170 & lacune & & $12 / 11 / 1386$ & 187 \\
\hline $21 / 11 / 1384$ & 164 & $22 / 1 / 1386$ & 151 & $19 / 11 / 1386$ & 159 \\
\hline $28 / 11 / 1384$ & 78 & $29 / 1 / 1386$ & 135 & $26 / 11 / 1386$ & 260 \\
\hline $5 / 12 / 1384$ & 118 & $5 / 2 / 1386$ & & $4 / 12 / 1386$ & 192 \\
\hline $12 / 12 / 1384$ & 78 & $12 / 2 / 1386$ & 133 & $10 / 12 / 1386$ & 264 \\
\hline $19 / 12 / 1384$ & 60 & $19 / 2 / 1386$ & 125 & \begin{tabular}{|l}
$17 / 12 / 1386$ \\
\end{tabular} & 279 \\
\hline $25 / 12 / 1384$ & 34 & $26 / 2 / 1386$ & 139 & $24 / 12 / 1386$ & 196 \\
\hline $2 / 1 / 1385$ & 38 & $5 / 3 / 1386$ & 130 & $31 / 12 / 1386$ & 219 \\
\hline $9 / 1 / 1385$ & 72 & $12 / 3 / 1386$ & 148 & $7 / 1 / 1387$ & 262 \\
\hline $16 / 1 / 1385$ & 55 & $19 / 3 / 1386$ & 182 & $14 / 1 / 1387$ & 214 \\
\hline $23 / 1 / 1385$ & 45 & $26 / 3 / 1386$ & 270 & $21 / 1 / 1387$ & 258 \\
\hline $30 / 1 / 1385$ & 65 & $2 / 4 / 1386$ & 245 & $28 / 1 / 1387$ & 131 \\
\hline $6 / 2 / 1385$ & 108 & $9 / 4 / 1386$ & 227 & $4 / 2 / 1387$ & 182 \\
\hline $13 / 2 / 1385$ & 101 & $16 / 4 / 1386$ & 186 & & \\
\hline
\end{tabular}

Tableau 12: Dépenses hebdomadaires (en livres tournois) dans les comptes d’Étienne Gervais, payeur des chantiers du palais et du château de Poitiers (1383-1387).

Malgré deux interruptions dans la documentation, la mise en graphique (histogramme) des dépenses hebdomadaires montre très bien la forte progression du chantier de Poitiers de 1383 et 1387. Il s'agit des sommes engagées dans les travaux conduits en régie. Les dépenses pour les travaux baillés à tâche étant soldées en fin d'année, elles n'ont pu être entrées dans ce tableau. On note néanmoins certaines phases importantes du chantier. En novembre 1384, Jean de Berry décide de donner une nouvelle impulsion au chantier en ajoutant la réfection de la tour Maubergeon (palais) à celle du château. Des ouvriers provenant de Mehun-sur-Yèvre viennent renforcer les équipes en place. On travaille non seulement à la préparation des gabarits mais aussi aux futurs dépôts de matériaux installés aux alentours du palais et à la transformation de l'hôtel de Vivonne (Poitiers) en fabrique de carreaux. Les dépenses hebdomadaires s'accroissent encore l'année suivante avec la reprise des fondations de la tour. Bien que le graphique semble montrer une progression constante, on peut 
noter de fortes variations dues essentiellement aux dépenses communes (matériaux, transports, outils, etc.).

\section{Conclusion}

Depuis la fin du XIX ${ }^{\mathrm{e}}$ siècle, les monographies consacrées à Jean de Berry confrontent les sources comptables, sans critique préalable, aux observations faites sur le terrain. La comptabilité de chantier vient simplement étayer les hypothèses formulées ou permet de retracer l'histoire d'un bâtiment disparu. Les comptes d'œuvre ne sont ni analysés ni présentés dans leur ensemble : ils apparaissent au chercheur comme un fonds documentaire aux origines incertaines et aux limites floues. Ce manque d'attention prêtée aux sources conduit à deux phénomènes : une méconnaissance des sources comptables et des exploitations incomplètes voire erronées. L'étude détaillée de la comptabilité de chantier ne permet pas seulement de vérifier les faits, elle enrichit considérablement nos connaissances car elle donne de très précieuses indications sur l'organisation, la conduite et le financement des travaux. De récentes études sur la maîtrise d'ouvrage à la fin du Moyen Âge ont largement exploité les sources comptables dans ce sens (voir les travaux de Philippe Bernardi, Florian Meunier, Étienne Hamon, Isabelle Chave, Denis Cailleaux, Jean Chapelot, etc.).

L'établissement d'une typologie des sources comptables de Jean de Berry permet en grande partie de comprendre l'organisation financière des chantiers du prince. Elle permet également d'ouvrir la recherche vers d'autres sources que les seuls comptes d'œuvres et, par ce biais, d'envisager l'étude de la maîtrise d'ouvrage du duc de Berry dans son acception la plus large. Cet élargissement du champ d'investigation a montré que l'organisation des chantiers et les circuits financiers ne s'appuient pas seulement sur l'administration des œuvres mise en place par le prince. Il montre également que les grands programmes résidentiels n'ont constitué qu'une partie, certes prépondérante, des travaux à mettre au crédit du duc de Berry. Enfin, il a également permis l'élargissement la recherche archivistique et la découverte de documents inédits, notamment dans les fonds de la BnF.

L'étude des comptes d'œuvres royaux et princiers de la fin du XIV siècle se trouve confrontée à un évident problème de conservation des comptes et des pièces justificatives produites sur les chantiers. Les monographies qui peuvent s'appuyer sur un corpus complet de comptes, comme ceux de la fabrique de Milan, sont rares: l'attribution du chantier de la Sainte-Chapelle de Vincennes au maître d'œuvre du roi, Raymond Du Temple, a pu être récemment établie par la découverte de deux feuillets provenant d'une comptabilité disparue ${ }^{109}$. Dans le cas de la maîtrise d'ouvrage de Jean de Berry, notre estimation montre que nous disposons d'environ une épave de compte sur dix et une pièce justificative sur 15000 . Les comptes des plus grands chantiers du prince ont de surcroît entièrement disparu. Dans un tel contexte, il n'est pas possible d'utiliser et d'exploiter ces sources sans apparat critique: l'historien de

109. Chapelot, J., Chapelot, O., Foucher, 2001, p. 433-488. 
la construction doit aborder l'exploitation des archives comptables avec les mêmes précautions qui s'imposent face à une chronique ou un texte littéraire. L'objet de ce travail préalable est de corriger les effets de distorsion produits par une documentation lacunaire et d'exploiter l'ensemble des données rescapées. Les lacunes causées par la dispersion ou la disparition des feuillets, des comptes entiers et des pièces justificatives cachent un très grand nombre d'informations. Une des lacunes les plus délicates est celle concernant les travaux baillés à tâche : leur témoignage a été perdu pour plus de la moitié des comptes. Ils représentaient pourtant une part importante, parfois la plus importante, de l'activité sur les chantiers. On imagine alors la prudence qu'il convient d'observer dans l'exploitation du texte et l'utilisation des sommes indiquées dans les comptes.

En outre, l'étude et la reconstitution des épaves de comptes nécessitent le recours à la codicologie. Elle n'est pas encore systématiquement utilisée dans les études des comptes d'œuvres; elle a pourtant montré son intérêt et l'importance de ses apports pour les deux feuillets de la Sainte-Chapelle de Vincennes ${ }^{110}$. Dans le cas de Jean de Berry, elle a permis la reconstitution de trois comptes dont les épaves avaient été dispersées. Elle a également permis de mettre en évidence des lacunes et la disparition de certains feuillets dans les comptes.

Jusqu'à présent, l'image d'un Jean de Berry mécène et ami des artistes s'imposait comme un postulat préalable à toute recherche, y compris pour l'histoire de ses constructions. L'apport de l'étude des sources comptables a permis l'émergence d'une nouvelle représentation de la maîtrise d'ouvrage du prince, mais aussi des moyens financiers qui ont pu concrétiser ses projets. Cette nouvelle représentation, moins centrée sur le personnage (mais sans en dénier le rôle ni l'implication), a mis au jour une réalité plus complexe impliquant l'ensemble de l'administration de Jean de Berry et mobilisant tous les moyens financiers dont elle disposait.

\section{Bibliographie non exhaustive concernant la maîtrise d'ouvrage de Jean de Berry ainsi que l'étude des sources comptables et financières}

AuTRAnd, Françoise, Jean de Berry, Paris, Fayard, 2000, 552 p.

BoN, Philippe, Les premiers "bleus" de France. Les carreaux de fä̈ence au décor peint fabriqué pour le duc de Berry, Mehun-sur-Yèvre, GHAMY, 1992, 335 p.

CHAMPEAUX, Alfred (de) et GAUCHERY, Paul, Les travaux d'art exécutés pour Jean de France, duc de Berry, avec une étude biographique des artistes employés par ce prince, Paris, H. Champion, 1894, 231 p.

GuifFreY, Jules (éd.), Inventaire de Jean Duc de Berry (1401-1416), 2 vol., Paris, 1894.

LeHOux, Françoise, Jean de France, duc de Berri. Sa vie, son action politique (1340-1416), Paris, A. Picard, 1966-1968, 4 vol.

110. Ibidem 
Magne, Lucien, Le palais de justice de Poitiers, étude de l'art français du XIVe siècle, Paris, Librairie centrale des Beaux-Arts, 1904, 172 p.

RAYNAUD, Clémence, «Ad instar capelle regie parisiensis : la Sainte Chapelle de Bourges, le grand dessein du duc de Berry », Bulletin Monumental, t. 162-4, 2004, p. 289-302.

TeYsSOT, Josiane, Riom capitale et bonne ville d'Auvergne, 1212-1557, Nonette, Créer, 1999, 428 p.

La Sainte-Chapelle de Bourges, une fondation disparue de Jean de France, duc de Berry, catalogue, Béatrice de CHANCEL-BARDELOT et Clémence RAYNAUD (dir.), Paris, Somogy, Bourges, Musée du Berry, 2004, 222 p.

\section{Travaux universitaires concernant la maîtrise d'ouvrage de Jean de Berry}

RAYNAUD, Clémence, La Sainte-Chapelle de Bourges, une fondation de Jean de Berry, Mémoire de troisième cycle d'histoire de l'art, École du Louvre, 1996, 2 vol.

SANCHEZ, Pierre, Trois chantiers de construction de Jean de France, duc de Berry et d'Auvergne, en son apanage d'Auvergne: le palais de Riom, les châteaux de Nonette et d'Usson. Étude de l'organisation des chantiers et des vestiges du décor sculpté des chapelles de Riom et de Nonette, Mémoire de maîtrise d'histoire de l'art, Paris I, 1993.

\section{Ouvrages concernant les finances à la fin du XIV siècle, l'approche des sources comptables et la codicologie}

CHAPELOT, Jean, «Charles V maître d'ouvrage: à propos de la construction du donjon de Vincennes et de quelques chantiers contemporains», in $\mathrm{Du}$ projet au chantier, maîtres d'ouvrages et maîtres d'œuvre aux XIVe-XVe siècles, Odette CHAPELOT (dir.), Paris, École des hautes études en sciences sociales, 2001, p 339-403.

CHAPELOT, Jean, CHAPELOT, Odette, FOUCHER, Jean-Pascal, «Un chantier et son maître d'œuvre: Raymond Du Temple et la Sainte-Chapelle de Vincennes en 1395-1396 », in Du projet au chantier, maîtres d'ouvrage et maîtres d'œuvre aux $X I V^{e}-X V I^{e}$ siècles, Odette CHAPELOT (dir.), Paris, École des hautes études en sciences sociales, 2001, p. 433-488.

JAQUIER, Élizabeth, «La politique castrale des Valois de Bourgogne dans les bailliages méridionaux de leur duché" in Du projet au chantier, maîtres d'ouvrage et maîtres d'œuvre aux XIVe-XVIe siècles, CHAPELOT (dir.), Paris, École des hautes études en sciences sociales, 2001, p. 154.

JASSEMIN, Henri, La Chambre des comptes de Paris au XVe siècle, Thèse pour le doctorat es lettres présentée à la faculté des lettres de l'Université de Paris, Paris, 1933. 
LEMAIRE, Jacques, Introduction à la codicologie, Louvain-La-Neuve, Institut d'études médiévales de l'Université catholique de Louvain, 1989, XI-265 p.

NORTIER, Michel, « Le sort des archives dispersées de la Chambre des comptes de Paris », BEC, t. 123, deuxième livraison, juillet-décembre 1965, p. 460-537.

REY, Maurice, Les finances royales sous Charles VI, les causes du déficit, 1388-1413, Paris, SEVPEN, 1965, 685 p.

REY, Maurice, Le domaine du roi et les finances extraordinaires sous Charles VI, 1388-1413, Paris, SEVPEN, 1965, 447 p.

TAVEAU-LaunaY, Isabelle, «Raymond Du Temple, maître d'œuvre des rois de France et des princes", in Du projet au chantier, maîtres d'ouvrage et maîtres d'œuvre aux XIV ${ }^{e}-X V I^{e}$ siècles, Odette CHAPELOT (dir.), Paris, École des Hautes Études en Sciences sociales, 2001, p. 323-338. 